\title{
In-flight calibration of Metis coronagraph on board of Solar Orbiter
}

\section{A. Liberatore, S. Fineschi, M. Casti, G. Capobianco, M. Romoli, et al.}

A. Liberatore, S. Fineschi, M. Casti, G. Capobianco, M. Romoli, V. Andretta, A. Bemporad, V. Da Deppo, Y. De Leo, M. Fabi, F. Frassetto, C. Grimani, K. Heerlein, P. Heinzel, G. Jerse, F. Landini, E. Magli, G. Naletto, G. Nicolini, M. Pancrazzi, M. G. Pelizzo, P. Romano, C. Sasso, A. Slemer, D. Spadaro, T. Straus, R. Susino, L. Teriaca, M. Uslenghi, C. A. Volpicelli, P. Zuppella, "Inflight calibration of Metis coronagraph on board of Solar Orbiter," Proc. SPIE 11852, International Conference on Space Optics - ICSO 2020, 1185248 (11 June 2021); doi: 10.1117/12.2599646 SPIE. Event: International Conference on Space Optics - ICSO 2021, 2021, Online 


\section{International Conference on Space Optics-ICSO 2020}

Virtual Conference

30 March-2 April 2021

Edited by Bruno Cugny, Zoran Sodnik, and Nikos Karafolas
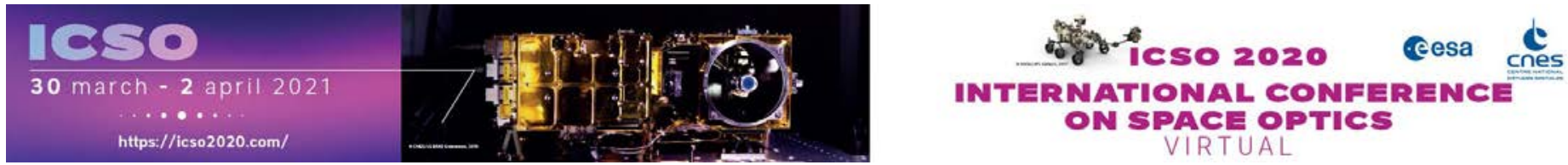

\section{In-flight calibration of Metis coronagraph on board of Solar Orbiter}

\section{Cesa ksonecatang ecnes}




\title{
In-flight calibration of Metis coronagraph on board of Solar Orbiter
}

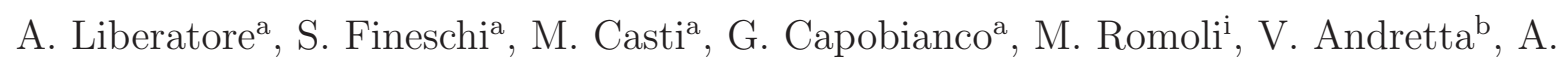

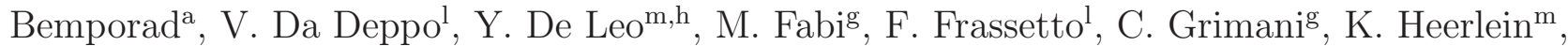
P. Heinzel ${ }^{f}$, G. Jerse ${ }^{c}$, F. Landini ${ }^{a}$, E. Magli ${ }^{k}$, G. Naletto ${ }^{j}$, G. Nicolini ${ }^{a}$, M. Pancrazzi ${ }^{a}$, M.G. Pelizzo $^{1}$, P. Romano ${ }^{d}$, C. Sasso ${ }^{b}$, A. Slemer ${ }^{1}$, D. Spadaro ${ }^{d}$, T. Straus ${ }^{b}$, R. Susino ${ }^{\mathrm{a}}$, L. Teriaca ${ }^{\mathrm{m}}$, M. Uslenghie , C.A. Volpicellia ${ }^{\mathrm{a}}$, and P. Zuppella ${ }^{\mathrm{l}}$

${ }^{a}$ INAF - Osservatorio Astrofisico di Torino, Italy

${ }^{\mathrm{b}}$ INAF - Osservatorio Astronomico di Capodimonte, Napoli, Italy

${ }^{\mathrm{c}} \mathrm{INAF}$ - Osservatorio Astronomico di Trieste, Italy

${ }^{\mathrm{d}} \mathrm{INAF}$ - Osservatorio Astrofisico di Catania, Italy

eINAF - IASF, Milano, Italy

${ }^{\mathrm{f}}$ Academy of Science, Astronomical Institute, Ondrejov, Czech Republic

gUniversità degli Studi di Urbino Carlo Bo and INFN, Firenze, Italy

${ }^{\mathrm{h}}$ Università di Catania, Italy

${ }^{i}$ Università di Firenze, Italy

jUniversità di Padova, Italy

${ }^{k}$ Politecnico di Torino, Italy

${ }^{\mathrm{l}} \mathrm{CNR}$ - IFN, Padova, Italy

mMPS, Göttingen, Germany

\begin{abstract}
Metis coronagraph is one of the remote-sensing instruments of the Solar Orbiter mission launched at the begin of 2020. The mission profile will allow for the first time the remote-sensing observation of the Sun from a very close distance and increasing the latitude with respect to the ecliptic plane. In particular, Metis is aimed at the overall characterization and study of the solar corona and solar wind. Metis instrument acquires images of the solar corona in two different wavelengths simultaneously; ultraviolet (UV) and visible-light (VL). The VL channel includes a polarimeter with an electro-optically modulating Liquid Crystal Variable Retarder (LCVR) to measure the linearly polarized brighness $\mathrm{pB}$ ) of the K-corona. This paper presents part of the in-flight calibration results for both wavelength channels together with a comparison with on-ground calibrations. The orientation of the K-corona linear polarization was used for the in-flight calibration of the Metis polarimeter. This paper describes the correction of the on-ground VL vignetting function after the in-flight adjustment of the internal occulter. The same vignetting function was adaptated to the UV channel.
\end{abstract}

Keywords: Solar Orbiter, Metis, Sun, coronagraph, solar corona, polarization, liquid crystal variable retarder.

\section{INTRODUCTION}

The Sun is surrounded by a million-degree atmosphere that extends into the solar system; the solar corona. The interaction between plasma and magnetic fields of the solar corona drives the physical phenomena that take place in the heliosphere and that affect the Earth magnetosphere. The diagnostics of the physical parameters of

Further author information: (Send correspondence to A.A.A.)

A.A.A.: E-mail: alessandro.liberatore@inaf.it, Telephone: (+39) 3473689597

B.B.A.: E-mail: silvano.fineschi@inaf.it, Telephone: (+39) 0118101919 
the coronal magnetized plasma are a crucial tool to understanding those phenomena. For this reason, more and more space missions aim to take data about our star. Among these, there is the Solar Orbiter mission.

Solar Orbiter (SolO), launched from Cape Canaveral in February 10 $0^{\text {th }} 2020$ at 04:03 UTC aboard a NASAprovided Atlas V 411 launch vehicle, is an ESA-led mission, with strong NASA participation, that will allow for the first time the remote-sensing observation of the Sun from as close as $0.28 \mathrm{AU}$. Moreover, this spacecraft (S/C) will be progressively more inclined to the ecliptic plane (until an ecliptic latitudes as high as $30^{\circ}$ ) observing, for the first time, the polar regions of the Sun. ${ }^{1}$ On board SolO there are 6 remote-sensing instruments and 4 sets of in-situ instruments for a total of 10 different experiments (see Fig. 1). The goal of the mission is to perform a detailed measurements of the inner heliosphere and solar wind by combining observations from all these instruments. Among the SolO remote-sensing instruments, there is the coronagraph Metis ${ }^{\dagger}$.

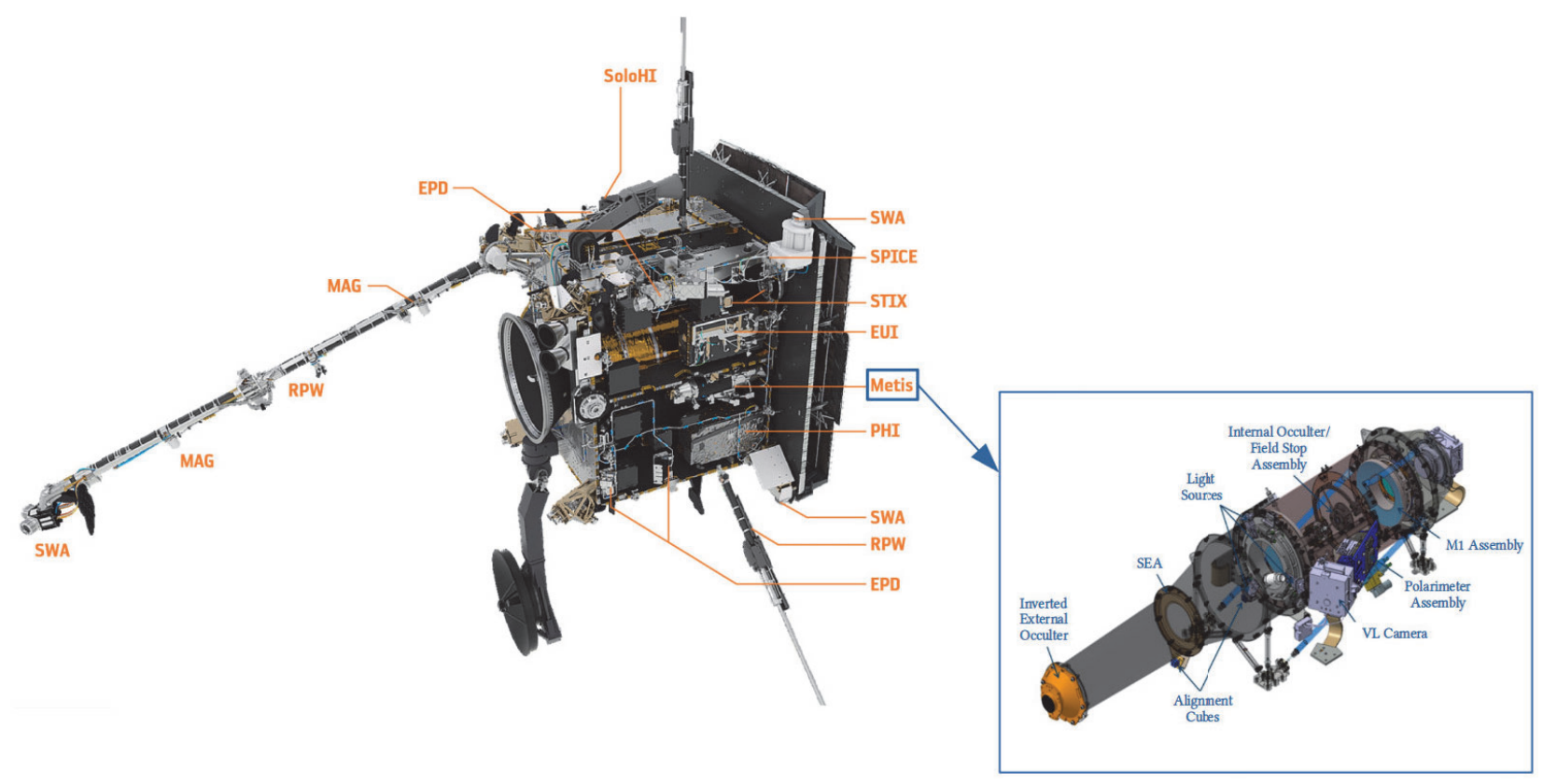

Figure 1: Solar Orbiter payload. ${ }^{1}$ Among the instruments on board the spacecraft, there is the Metis coronagraph. A $3 \mathrm{D}$ CAD of Metis is show in the blue rectangle.

Metis instrument is a multi-wavelength externally occulted coronagraph. ${ }^{2}$ It acquires images of the solar corona in two different wavelengths simultaneously: ultraviolet (UV) and visible light (VL). The UV channel works on the narrow-band HI Ly- $\alpha, 121.6 \mathrm{~nm}$, while the VL one works on the broadband 580-640 nm (Fig. 2).

One of the science objectives of Metis coronagraph is to study the Kontinuierlich corona ("K-corona") evaluating, for example, the electron density in order to characterize the coronal plasma and to determine the origin and acceleration of the fast and slow solar wind. The K-corona, generated by the Thomson scattering of the photospheric light from free coronal electrons, is linearly polarized. ${ }^{3,4}$ In particular, it is well known that the K-corona brightness is directly proportional to the electron column density along the line of sight (LoS). Then, by measuring the brightness and adopting proper geometrical assumptions, it is possible to estimate the electron density. ${ }^{5}$ However, being the coronal emission optically thin, this kind of observations will contain not only the $\mathrm{K}$-corona but also a component due to the scattering of photospheric light from dust as well as a stray light component. In order to eliminate these components, Metis will measure the polarized brightness (pB) -defined as the difference between the tangential and the radial polarization components-. To do that, along the optical path of the visible channel, a polarimeter assembly with the presence of an electro-optically modulating Liquid Crystal Variable Retarder (LCVR) gives the possibility to measure the linear polarization of the K-corona

\footnotetext{
${ }^{\dagger}$ In ancient Greek mythology, Metis was the first wife of Zeus and symbol of wisdom and deep thought.
} 
(through the acquisition of 4 images with different angle of polarizations) and then the coronal electron density can be derived.

More details on the Metis optical design and characteristics are described in the following. In particular, in Section 2, the expected instrument optical performances and the instrument internal structure are provided. Inter alia, more details on the LCVR into the Metis polarimeter are reported in Subsection 2.1 and a summary about the on-ground calibration is given in Subsection 2.2. Moving to Section 3, a detailed description of the preliminary in-flight calibration, showing problems encountered and solutions adopted, is presented. Finally, the conclusions (Section 4) based on the obtained results.

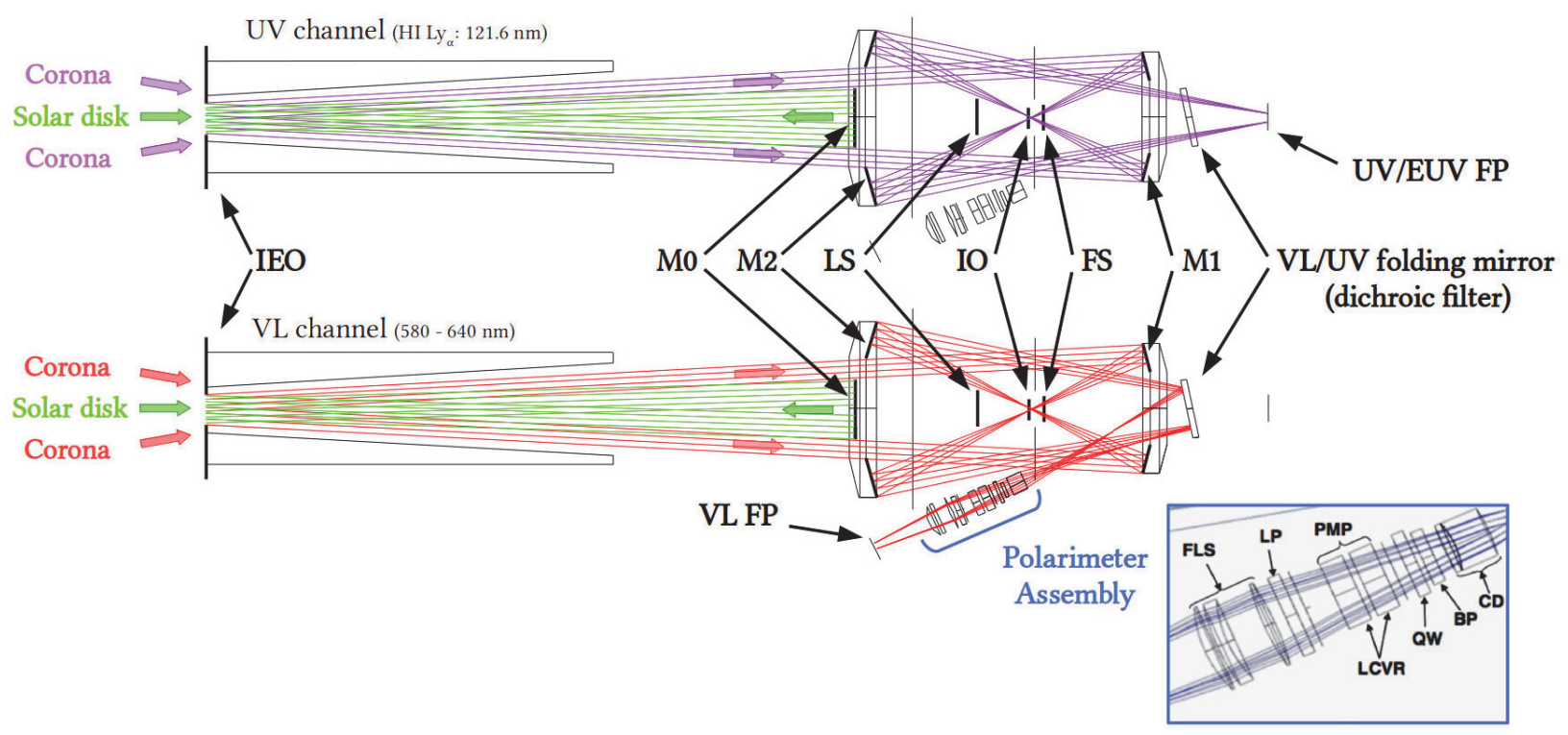

Figure 2: Metis ray trace for he UV and VL channels with a zoom on the VL polarimeter assembly.

\section{METIS CORONAGRAPH}

Due to the Solar Orbiter wide range of distances from the Sun, the on-board instruments need a particular design to be able to be operational in classical space condition but also to sustain the high thermal flux at the perihelion. For this reason, the Metis occultation scheme is based on an "inverted" external-occulter (IEO) to reduce the high thermal load (temperatures higher than $400^{\circ} \mathrm{C}$ ) on the instrument when the spacecraft is nearest to the Sun. In fact, in a classical externally-occulted coronagraph, the disk-light rejecting mirror is much larger than the circular one used in the inverted-occultation scheme (M0 in Fig. 2). This reduces by two orders of magnitude the thermal load on the rejection mirror. ${ }^{6}$ In particular, the IEO consists of a circular aperture $(\varnothing 40.0 \mathrm{~mm})$ at the narrow end of a truncated cone (the "boom"). The disk-light is rejected back by a spherical heat-rejection mirror M0 $(\varnothing 71.0 \mathrm{~mm})$ distant $800 \mathrm{~mm}$ from the IEO. The coronal light, is instead collected by an on-axis aplanatic Gregorian telescope. The suppression of the diffracted light off the edges of the IEO and M0 is achieved, respectively, with an internal occulter (IO) and a Lyot trap (LS). ${ }^{7,6}$

The primary (M1) and secondary (M2) telescope mirrors have a $\mathrm{Al} / \mathrm{MgF}_{2}$ coating to enhanced reflectivity at $\mathrm{HI} 121.6 \mathrm{~nm}\left(\mathrm{Ly}_{\alpha}\right)$ and has an high reflectivity also the visible wavelength range $580-640 \mathrm{~nm}$. After these reflections, the coronal light is then splitted by an UV interference filter $\left(12^{\circ}\right.$ angle of inclination with respect to the optical axis) that works by selecting the $121.6 \mathrm{~nm}$ UV band in transmission and reflecting the VL to the polarimeter. Is here, inside the polarimeter, that a broadband filter selects the VL bandpass 580-640 nm. The coronal light, entering into the polarimeter, sequentially encounters the following elements: a collimating doublet (CD), the VL bandpass filter (BP), focus lens system (FLS), a quarter-wave (QW) plate retarder, a polarisation modulation package (PMP) composed of two liquid crystals variable retarders (LCVRs) -more information in 
the next subsection-, a linear polariser (LP) and a focus lens system (FLS). A detail of the Metis polarimeter is shown in the blue rectangle in Fig. 2.

Finally, on the focal planes of the two optical paths, there are the UV and VL detectors. The platescale of the UV and VL detectors are $20 \mathrm{arcsec} /$ pixel and $10 \mathrm{arcsec} /$ pixel respectively. All these, and others, optical performances are summarized in Tab. 1. More detailed opto-mechanical information can be found in Ref,. 6, 7.

Table 1: Main optical performances of Metis instrument. ${ }^{7,6}$

\begin{tabular}{|c|c|}
\hline \multicolumn{2}{|l|}{ Spectral range } \\
\hline Multi-wavelength & $\begin{array}{l}\text { VL: } 580-640 \mathrm{~nm} \\
\text { UV: } \mathrm{HI} \mathrm{Ly} \mathrm{Ly}_{\alpha}, 121.6 \pm 7.5 \mathrm{~nm}(\mathrm{FWHM})\end{array}$ \\
\hline \multicolumn{2}{|l|}{ Telescope } \\
\hline Type & (Inverted) Externally occulted, on-axis aplanatic Gregorian \\
\hline Overall length & $1309 \mathrm{~mm}$ \\
\hline Effective focal length & $\begin{array}{l}\text { VL: } 200 \mathrm{~mm} \\
\mathrm{UV}: 300 \mathrm{~mm}\end{array}$ \\
\hline Inverted External Occulter (IEO) & Circular aperture: $\varnothing 40.0 \mathrm{~mm}$ \\
\hline Distance IEO-M0 (boom) & $800.0 \mathrm{~mm}$ \\
\hline Sun-light rejection mirror (M0) & $\begin{array}{l}\text { Spherical: } \varnothing 71.0 \mathrm{~mm} \\
\text { Curvature radius: } 1600.0 \mathrm{~mm}\end{array}$ \\
\hline Primary mirror (M1) & $\begin{array}{l}\text { On axis ellipsoidal: outer } \varnothing 160.0 \mathrm{~mm} \text {, inner } \varnothing 88.0 \mathrm{~mm} \\
\text { Curvature radius: } 272.0 \mathrm{~mm} \\
\text { Conic: }-0.662\end{array}$ \\
\hline Secondary mirror (M2) & $\begin{array}{l}\text { On axis ellipsoidal: outer } \varnothing 216.0 \mathrm{~mm} \text {, inner } \varnothing 125.0 \mathrm{~mm} \\
\text { Curvature radius: } 312.4 \mathrm{~mm} \\
\text { Conic: }-0.216\end{array}$ \\
\hline Internal occulter (IO) & $\begin{array}{l}\text { Distance M1-IO: } 154.8 \mathrm{~mm} \\
\text { Circular: } \varnothing 5.0 \mathrm{~mm}\end{array}$ \\
\hline \multicolumn{2}{|l|}{ Detectors } \\
\hline Plate scale & $\begin{array}{l}\text { VL: } 10 \operatorname{arcsec} / \text { pixel } \\
\text { UV: } 20 \operatorname{arcsec} / \text { pixel }\end{array}$ \\
\hline Image size & $\begin{array}{l}\text { VL: } 20.7 \mathrm{~mm}(2048 \times 2048) \text { with } 10 \mu \mathrm{m} \text { pixel size } \\
\text { UV: } 30.7 \mathrm{~mm}(1024 \times 1024) \text { with } 30 \mu \mathrm{m} \text { equivalent pixel size }\end{array}$ \\
\hline Field of view (FoV) & $\begin{array}{l}1.6^{\circ}-2.9^{\circ} \text { annular, off-limb corona } \\
1.7-3.1 R_{\odot} @ 0.28 \mathrm{AU} \\
3.0-5.5 R_{\odot} @ 0.5 \mathrm{AU}\end{array}$ \\
\hline
\end{tabular}

\subsection{Liquid Crystals Variable Retarders}

As explained in Section 1 and at the beginning of Section 2, along the optical path of the visible channel, just before the VL detector, is set a polarimeter assembly with the presence of a couple of electro-optically modulating Liquid Crystals Variable Retarders (LCVRs)..$^{8,9}$ 

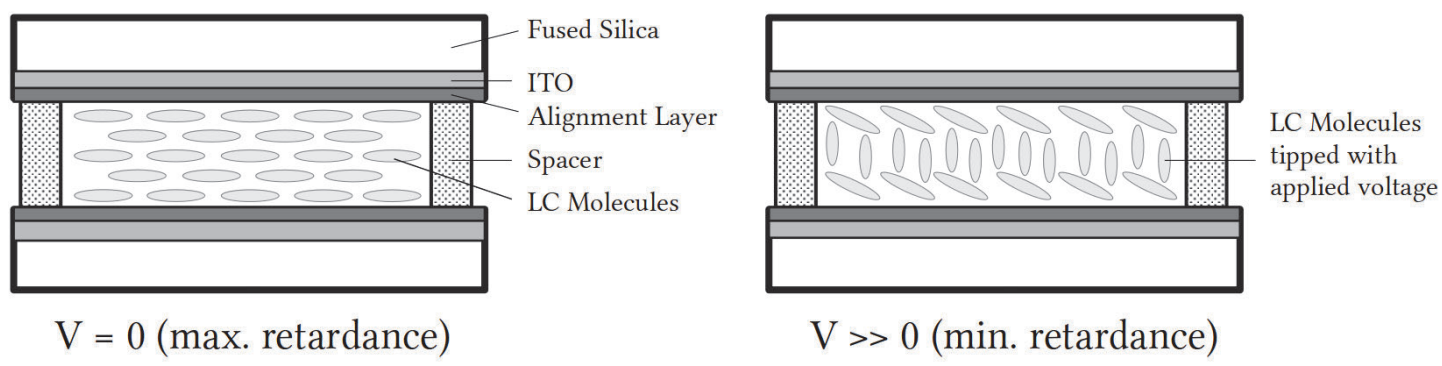

Figure 3: Schematic representation of a Liquid Crystal Variable Retarders operation.

The LCVRs of the Metis polarimeter consist of optically anisotropic liquid crystal molecules embedded between two glasses with a conductive film. These molecules have been accommodated in the cells with an ordered orientation and the effective birefringence of the cell can be changed by applying an electric field that rotates the molecules. Consequently, a change in orientation of the molecules introduce an optical retardance in the orthogonal polarization components of the incoming light. The advantages introduced by this technology are several. In this respect, retarders based on liquid crystals permits to avoid the use of mechanical parts, reducing noise, failure probability and mass. Moreover, the power consumption of the liquid crystal-based retarders is reduced and their response is very quick, providing a fast modulation of the polarization state of light. In the other hand, LCVR devices are sensitive to temperature: retardance depends on temperature cells. For this reason, Metis PMP has been provided with a temperature controller, in order to guarantee the temperature uniformity on liquid crystal cells. Furthermore, the LCVR cells that compose the Metis PMP have been assembled with the fast axes aligned and the pre-tilt angle of the liquid crystal molecules in opposite direction, in order to obtain a wider acceptance angle (that results to be equal to $\left.\pm 4^{\circ}\right) .{ }^{10,11}$

Obviously, to evaluate the effective polarization angle corrispondent to a certain applied tension, we have to consider the entire polarimeter assembly (which includes, in addition to the PMP, also a LP and a QW). In particular, in order to analyze the data acquired for the polarimetric VL channel calibration, a new reference frame has been introduced; the Polarimeter Instrument Level System (PILS) reference frame. ${ }^{11}$ Therefore, the on-ground data analysis is based on the PILS reference frame (Fig. 4) which is define as follows:

- $\mathrm{X}_{P I L S}=$ parallel to the incoming light direction, pointing the Metis VL detector;

- $\mathrm{Z}_{P I L S}=$ parallel to the Metis linear polarizer acceptance axis

- $\mathrm{Y}_{P I L S}=$ as consequence

Basically, the difference between the "standard" reference frame [i.e. as reported in literature] used to retrieve the Stokes vector reported in literature and our PILS reference frame is a $90^{\circ}$ rotation around the y-axis. This means that, for retrieving the theoretical Stokes vector in our case is necessary to apply a rotation matrix to them. Fig. 5 shows the two reference frames to be compared. In particular, as we will see in Subsec. 2.2, during on ground calibration we considered polarization angles equals to $0^{\circ}, 45^{\circ}, 90^{\circ}$ and $135^{\circ}$.

Looking at the PILS reference frame, we can define the Stokes parameters $\left(S_{0}, S_{1}, S_{2}\right)=(I, Q, U)$ as:

$$
\begin{aligned}
& I=\hat{n}_{Z P I L S}+\hat{n}_{Y P I L S} \\
& Q=\hat{n}_{Z P I L S}-\hat{n}_{Y P I L S} \\
& U=\left(\hat{n}_{Z P I L S}+\hat{n}_{Y P I L S}\right)-\left(-\hat{n}_{Z P I L S}+\hat{n}_{Y P I L S}\right)
\end{aligned}
$$




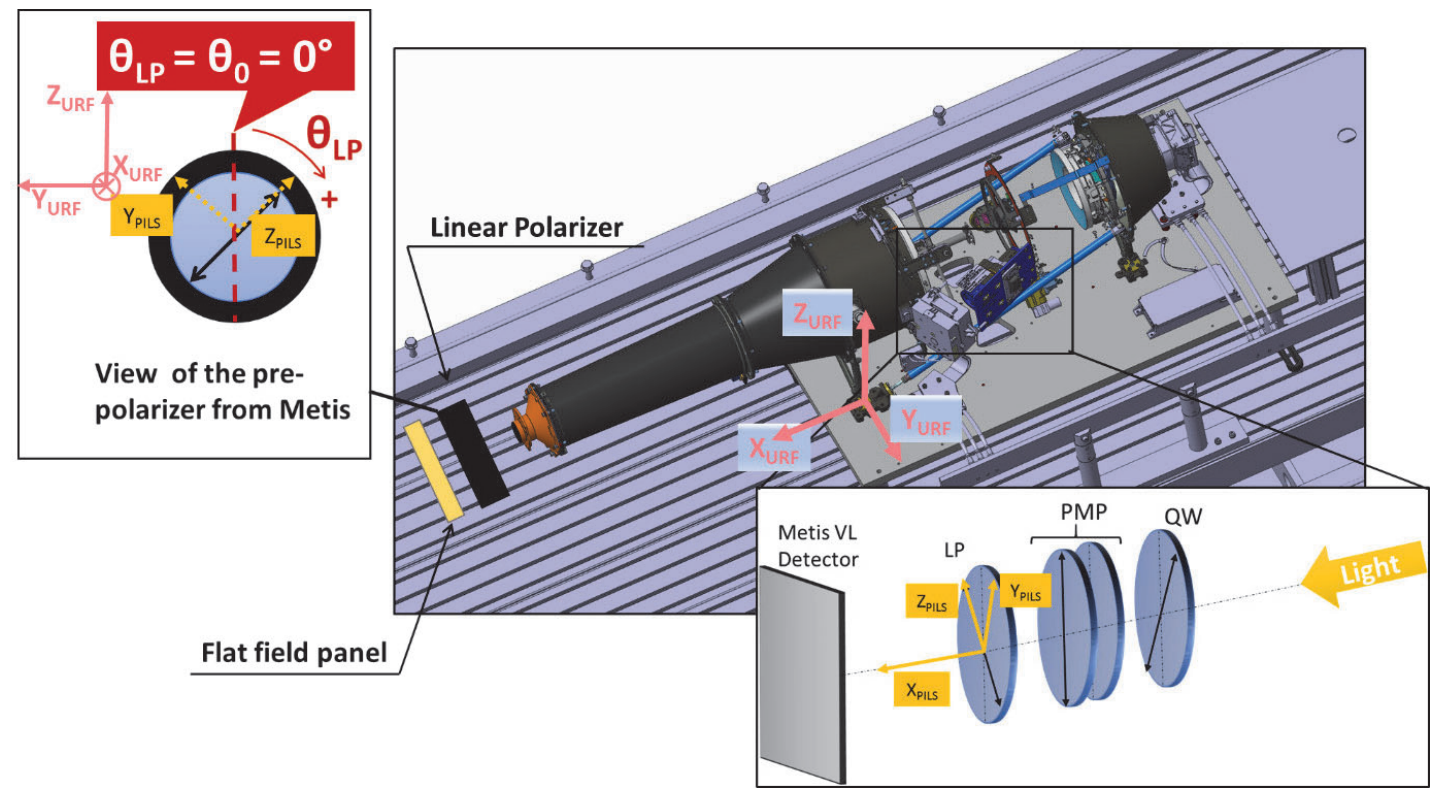

Figure 4: Schematic view of the set up used for data acquisition and Metis polarimeter optical elements relative orientations in PILS reference frame [compared with the Unit Reference Frame (URF)]. The arrows in the optical elements represent, respectively: the fast axes for both the QW and the LP, and the PMP polarization axis.

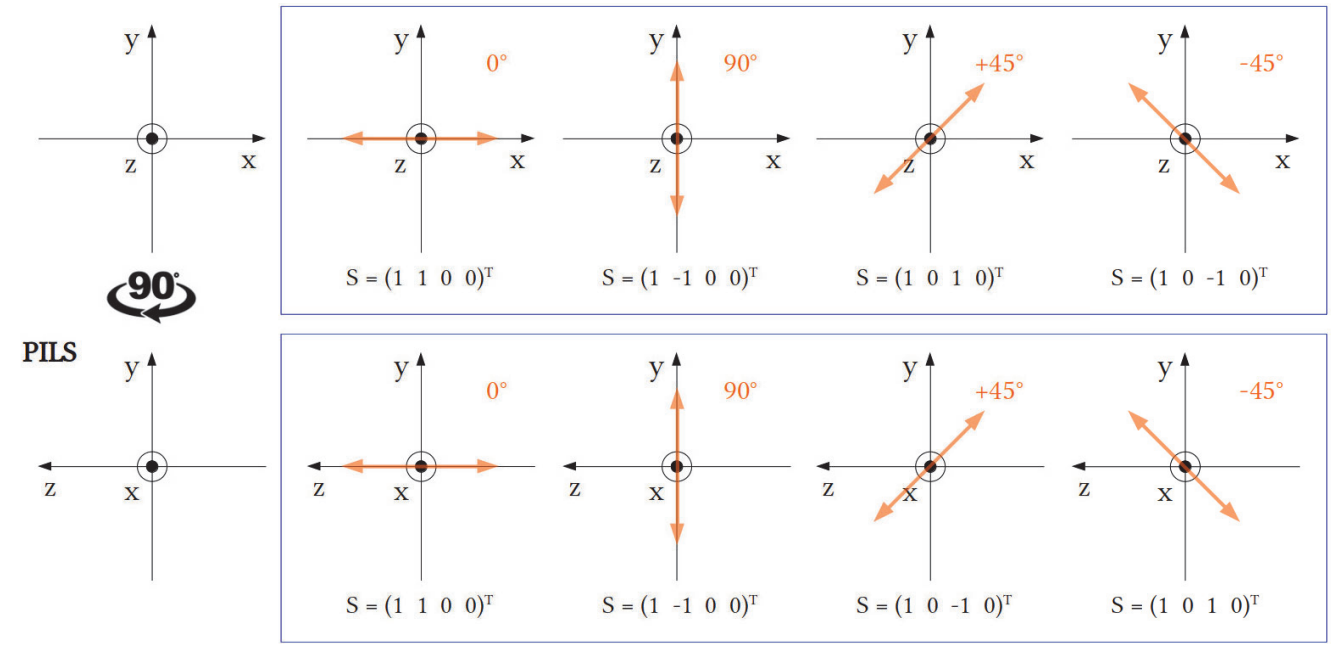

Figure 5: Stokes vectors for linearly polarized radiation in the PILS reference frame (bottom row) compared to the "standard" one (upper row).

\subsection{On-ground calibration}

Being this paper specifically dedicated to in-flight calibration, not many details will be provided about onground one. Anyway, some information can be useful to the fully understanding of the in-flight calibration and, if necessary, more information about on-ground calibration can be found in Refs. 6, 11 and 12.

Part of the Metis on-ground calibration aims at characterizing the polarimetric response in visible light. This goal is achieved by retrieving the Metis polarimeter demodulation tensor $\mathbf{X}^{\dagger}$ (obtained by computing and inverting the modulation matrix $\mathbf{X}$ associated with each pixel of the acquired polarimetric images). This tensor will allow us to polarimetrically characterize the incoming light from the intensity. A full polarimetric characterization requires to find the Stokes vector $\mathbf{S}=\left(S_{0}, S_{1}, S_{2}, S_{3}\right)=(I, Q, U, V)$. Being the $S_{3}(\equiv V)$ 
parameter associated with circular polarization, we are interested just in the first three Stokes parameters (for the study of the linear polarization present in the solar corona due to the Thomson scattering - see Sec. 1). If we modulate the incoming light by varying the voltage applied to the LCVRs tacking four images $\mathbf{m}=$ $\left[m_{1}, m_{2}, m_{3}, m_{4}\right]$ at different retardance values and knowing the demodulation tensor, we can obtain the Stokes vector $\mathbf{S}$ of the incoming radiation by solving the linear system:

$$
\mathbf{m}=\mathbf{X S} \quad \rightarrow \quad \mathbf{S}=\mathbf{X}^{\dagger} \mathbf{m}
$$

The modulation matrix $\mathbf{X}$, as we are about to see, come from the Mueller matrix $\mathbf{M}_{P O L}$ associated with Metis polarimeter. In particular, it is obtained as the product of the Mueller matrices associated with the polarizing elements inside the polarimeter as follow:

$$
\begin{aligned}
\mathbf{M}_{P O L} & =\mathbf{M}_{L P}(\theta=0) \mathbf{M}_{P M P}\left(\theta=-\frac{\pi}{4}, \delta\right) \mathbf{M}_{Q W}\left(\theta=\frac{\pi}{2}\right) \\
& =\frac{1}{2}\left(\begin{array}{llll}
1 & 1 & 0 & 0 \\
1 & 1 & 0 & 0 \\
0 & 0 & 0 & 0 \\
0 & 0 & 0 & 0
\end{array}\right)\left(\begin{array}{cccc}
1 & 0 & 0 & 0 \\
0 & \cos \delta & 0 & -\sin \delta \\
0 & 0 & 1 & 0 \\
0 & \sin \delta & 0 & \cos \delta
\end{array}\right)\left(\begin{array}{cccc}
1 & 0 & 0 & 0 \\
0 & 1 & 0 & 0 \\
0 & 0 & 0 & 1 \\
0 & 0 & -1 & 0
\end{array}\right) \\
& =\frac{1}{2}\left(\begin{array}{cccc}
1 & \cos \delta & \sin \delta & 0 \\
1 & \cos \delta & \sin \delta & 0 \\
0 & 0 & 0 & 0 \\
0 & 0 & 0 & 0
\end{array}\right)
\end{aligned}
$$

where $\theta$ is the rotation angle of the linear polarization vector and $\delta=2 \theta$ the retardance. It is possible to see that the form obtained for Eq. 2 is equivalent to $\mathbf{M}_{P O L}=\mathbf{M}_{L P} \mathbf{M}_{r o t}(2 \theta=\delta)$ where $\mathbf{M}_{\text {rot }}$ is the Mueller matrix of a rotating element. More details and a step by step description about how to obtain $\mathbf{M}_{P O L}$ are shown in Ref. 11 .

The last column of the retrieved Mueller matrix is composed of elements equal to zero; this proves that this configuration does not suffer from the possible presence of circularly polarized radiation. Now it is possible to retrieve the Metis polarimeter modulation matrix. In fact, each row of this matrix corresponds to the first row of the Mueller matrix, assuming a different retardance angle and, consequently, a different applied voltage. Then, we have:

$$
\mathbf{X}=\frac{1}{2}\left(\begin{array}{ccc}
1 & \cos \delta_{1} & \sin \delta_{1} \\
1 & \cos \delta_{2} & \sin \delta_{2} \\
1 & \cos \delta_{3} & \sin \delta_{3} \\
1 & \cos \delta_{4} & \sin \delta_{4}
\end{array}\right)=\frac{1}{2}\left(\begin{array}{ccc}
1 & 1 & 0 \\
1 & 0 & 1 \\
1 & -1 & 0 \\
1 & 0 & -1
\end{array}\right)
$$

If we consider the optical retardance angles $\delta_{i}=0^{\circ}, 90^{\circ}, 180^{\circ}, 270^{\circ}$ (see Tab. 2), we can obtain a theoretical modulation matrix $\mathbf{X}$ that assumes the following values:

$$
\mathbf{X}=\frac{1}{2}\left(\begin{array}{ccc}
1 & 1 & 0 \\
1 & 0 & 1 \\
1 & -1 & 0 \\
1 & 0 & -1
\end{array}\right)
$$

Now we can retrieve the theoretical demodulation tensor $\mathbf{X}^{\dagger}$ as Moore-Penrose inverse of the theoretical modulation matrix $\mathbf{X}$ just found. What we obtain is:

$$
\mathbf{X}^{\dagger}=\frac{1}{2}\left(\begin{array}{cccc}
1 & 1 & 1 & 1 \\
2 & 0 & -2 & 0 \\
0 & 2 & 0 & -2
\end{array}\right)
$$


Then, the theoretical Stokes parameters can be easily obtained just solving Eq. 1:

$$
\left(\begin{array}{l}
I \\
Q \\
U
\end{array}\right)=\frac{1}{2}\left(\begin{array}{cccc}
1 & 1 & 1 & 1 \\
2 & 0 & -2 & 0 \\
0 & 2 & 0 & -2
\end{array}\right)\left(\begin{array}{l}
m_{1} \\
m_{2} \\
m_{3} \\
m_{4}
\end{array}\right)=\mathbf{X}^{\dagger}\left(\begin{array}{l}
m_{1} \\
m_{2} \\
m_{3} \\
m_{4}
\end{array}\right)
$$

However, with the intention of deriving the real demodulation tensor associated with the Metis polarimeter, during the on-ground calibration activities we acquired data related to different well-known polarized incoming light states and we modulated them by applying different voltages to the LCVR cells (the considered pre-pol. position are reported in Tab.2). For each pre-pol. position, the left part of Eq. 1 assumes the following expression:

$$
\left(\begin{array}{l}
\bar{m}_{1}^{x \text { deg }} \\
\bar{m}_{2}^{x \text { deg }} \\
\bar{m}_{3}^{x \text { deg }} \\
\bar{m}_{4}^{\text {xdeg }}
\end{array}\right)=\mathbf{X}\left(\begin{array}{l}
I^{x d e g} \\
Q^{x d e g} \\
U^{x d e g}
\end{array}\right)=\left(\begin{array}{lll}
x_{11} & x_{12} & x_{13} \\
x_{21} & x_{22} & x_{23} \\
x_{31} & x_{32} & x_{33} \\
x_{41} & x_{42} & x_{43}
\end{array}\right)\left(\begin{array}{l}
I^{x d e g} \\
Q^{x d e g} \\
U^{x d e g}
\end{array}\right)
$$

where $\mathrm{xdeg}=\left[-45^{\circ}, 0^{\circ}, 45^{\circ}, 90^{\circ}\right]$ is the angular position of the linear pre-polarizer acceptance axis referred to the PILS reference frame, and:

$$
\bar{m}_{i}^{x \operatorname{deg}}=\frac{m_{i}^{x \operatorname{deg}}}{\frac{1}{2} \sum_{i=1}^{4} m_{i}^{x d e g}}
$$

where $i$ the voltage applied to the Metis polarimeter corresponding to a polarimeter rotation angle $0^{\circ}, 45^{\circ}, 90^{\circ}$ and $135^{\circ}$ in this order.

Table 2: Positions of the Pre-Polarizer during data acquisition and the correspondent angles in the considered

\begin{tabular}{|c|c|c|}
\hline $\begin{array}{c}\text { Pre-Pol rotation angle } \\
\left(\text { wrt } \mathrm{URF}\left[\theta_{L P}=0 \text { when LP axis } / / z_{U R F}\right]\right)\end{array}$ & $\begin{array}{l}\text { xdeg [Pre-Pol angle] } \\
\text { (wrt PILS) }\end{array}$ & $\begin{array}{c}\text { Theoretical Stokes Vector } \\
\text { (wrt PILS) }\end{array}$ \\
\hline $0^{\circ}$ & $-45^{\circ}$ & $\left(\begin{array}{ll}1 & 0\end{array}\right.$ \\
\hline $45^{\circ}$ & $0^{\circ}$ & $\left(\begin{array}{ll}1 & 1\end{array}\right.$ \\
\hline $90^{\circ}$ & $45^{\circ}$ & $\left(\begin{array}{lll}1 & 0 & -1\end{array}\right)^{T}$ \\
\hline $135^{\circ}$ & $90^{\circ}$ & $\left(\begin{array}{ll}1 & -1\end{array}\right.$ \\
\hline
\end{tabular}
reference frame (PILS). In the last column, the associated theoretical Stokes vector. In this respect, it is necessary to analyze the Pre-Polarizer fast axis position with respect to the polarimeter analyzer, the PILS z-axis. ${ }^{11}$

To solve the linear system shown in Eq. 7, we need at least 12 equations (because we have 12 unknown quantities: $x_{i j}$ with $\left.1 \leq i, j \leq 4\right)$. In particular, this analysis is performed considering four different voltages

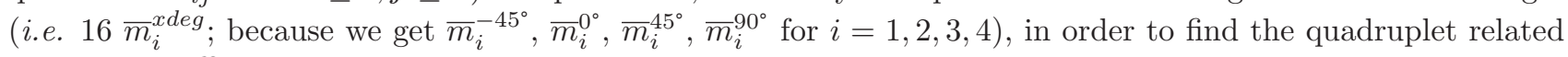
to the most efficient demodulation matrix.

The final form of the linear system is shown in Eq. 9 where $\bar{m}_{1}$ refers to measure performed when the rotation angle correspondent to the applied voltage is $\approx 0^{\circ} ; \bar{m}_{2}$ refers to measure performed when the rotation angle correspondent to the applied voltage is $\approx 45^{\circ} ; \bar{m}_{3}$ refers to measure performed when the rotation angle correspondent to the applied voltage is $\approx 90^{\circ}$ and $\bar{m}_{4}$ refers to measure performed when the rotation angle correspondent to the applied voltage is $\approx 135^{\circ}$. 


$$
\left(\begin{array}{c}
\bar{m}_{1}^{-45^{\circ}} \\
\bar{m}_{2}^{-45^{\circ}} \\
\bar{m}_{3}^{-45^{\circ}} \\
\bar{m}_{4}^{-45^{\circ}} \\
\bar{m}_{1}^{0^{\circ}} \\
\bar{m}_{2}^{0^{\circ}} \\
\bar{m}_{3}^{0^{\circ}} \\
\bar{m}_{4}^{0^{\circ}} \\
\bar{m}_{1}^{45^{\circ}} \\
\bar{m}_{2}^{45^{\circ}} \\
\bar{m}_{3}^{45^{\circ}} \\
\bar{m}_{4}^{45^{\circ}} \\
\bar{m}_{1}^{90^{\circ}} \\
\bar{m}_{2}^{90^{\circ}} \\
\bar{m}_{3}^{90^{\circ}} \\
\bar{m}_{4}^{90^{\circ}}
\end{array}\right)=\left(\begin{array}{ccccccccccccc}
I^{-45} & Q^{-45} & U^{-45} & 0 & 0 & 0 & 0 & 0 & 0 & 0 & 0 & 0 \\
0 & 0 & 0 & I^{-45} & Q^{-45} & U^{-45} & 0 & 0 & 0 & 0 & 0 & 0 \\
0 & 0 & 0 & 0 & 0 & 0 & I^{-45} & Q^{-45} & U^{-45} & 0 & 0 & 0 \\
0 & 0 & 0 & 0 & 0 & 0 & 0 & 0 & 0 & I^{-45} & Q^{-45} & U^{-45} \\
I^{0} & Q^{0} & U^{0} & 0 & 0 & 0 & 0 & 0 & 0 & 0 & 0 & 0 \\
0 & 0 & 0 & I^{0} & Q^{0} & U^{0} & 0 & 0 & 0 & 0 & 0 & 0 \\
0 & 0 & 0 & 0 & 0 & 0 & I^{0} & Q^{0} & U^{0} & 0 & 0 & 0 \\
0 & 0 & 0 & 0 & 0 & 0 & 0 & 0 & 0 & I^{0} & Q^{0} & U^{0} \\
I^{45} & Q^{45} & U^{45} & 0 & 0 & 0 & 0 & 0 & 0 & 0 & 0 & 0 \\
0 & 0 & 0 & I^{45} & Q^{45} & U^{45} & 0 & 0 & 0 & 0 & 0 & 0 \\
0 & 0 & 0 & 0 & 0 & 0 & I^{45} & Q^{45} & U^{45} & 0 & 0 & 0 \\
0 & 0 & 0 & 0 & 0 & 0 & 0 & 0 & 0 & I^{45} & Q^{45} & U^{45} \\
I^{90} & Q^{90} & U^{90} & 0 & 0 & 0 & 0 & 0 & 0 & 0 & 0 & 0 \\
0 & 0 & 0 & I^{90} & Q^{90} & U^{90} & 0 & 0 & 0 & 0 & 0 & 0 \\
0 & 0 & 0 & 0 & 0 & 0 & I^{90} & Q^{90} & U^{90} & 0 & 0 & 0 \\
0 & 0 & 0 & 0 & 0 & 0 & 0 & 0 & 0 & I^{90} & Q^{90} & U^{90} \\
x_{13} \\
x_{21} \\
x_{22} \\
x_{23} \\
x_{31} \\
x_{32} \\
x_{33} \\
x_{41} \\
x_{42} \\
x_{43}
\end{array}\right)
$$

Considering the elements of the theoretical Stokes vectors associated to the linear pre-polarizer position (Tab. 2) with respect to the PILS reference frame, is shown in Eq. 10. In order to compute the modulation matrix, Eq. 10 has been solved for each detector pixel.

$$
\left(\begin{array}{c}
\bar{m}_{1}^{-45^{\circ}} \\
\bar{m}_{2}^{-45^{\circ}} \\
\bar{m}_{3}^{-45^{\circ}} \\
\bar{m}_{4}^{-45^{\circ}} \\
\bar{m}_{1}^{0^{\circ}} \\
\bar{m}_{2}^{0^{\circ}} \\
\bar{m}_{3}^{0^{\circ}} \\
\bar{m}_{4}^{0^{\circ}} \\
\bar{m}_{1}^{45^{\circ}} \\
\bar{m}_{2}^{45^{\circ}} \\
\bar{m}_{3}^{45^{\circ}} \\
\bar{m}_{4}^{45^{\circ}} \\
\bar{m}_{1}^{90^{\circ}} \\
\bar{m}_{2}^{90^{\circ}} \\
\bar{m}_{3}^{90^{\circ}} \\
\bar{m}_{4}^{90^{\circ}}
\end{array}\right)=\left(\begin{array}{ccccccccccccc}
1 & 0 & 1 & 0 & 0 & 0 & 0 & 0 & 0 & 0 & 0 & 0 \\
0 & 0 & 0 & 1 & 0 & 1 & 0 & 0 & 0 & 0 & 0 & 0 \\
0 & 0 & 0 & 0 & 0 & 0 & 1 & 0 & 1 & 0 & 0 & 0 \\
0 & 0 & 0 & 0 & 0 & 0 & 0 & 0 & 0 & 1 & 0 & 1 \\
1 & 1 & 0 & 0 & 0 & 0 & 0 & 0 & 0 & 0 & 0 & 0 \\
0 & 0 & 0 & 1 & 1 & 0 & 0 & 0 & 0 & 0 & 0 & 0 \\
0 & 0 & 0 & 0 & 0 & 0 & 1 & 1 & 0 & 0 & 0 & 0 \\
0 & 0 & 0 & 0 & 0 & 0 & 0 & 0 & 0 & 1 & 1 & 0 \\
1 & 0 & -1 & 0 & 0 & 0 & 0 & 0 & 0 & 0 & 0 & 0 \\
0 & 0 & 0 & 1 & 0 & -1 & 0 & 0 & 0 & 0 & 0 & 0 \\
0 & 0 & 0 & 0 & 0 & 0 & 1 & 0 & -1 & 0 & 0 & 0 \\
0 & 0 & 0 & 0 & 0 & 0 & 0 & 0 & 0 & 1 & 0 & -1 \\
1 & -1 & 0 & 0 & 0 & 0 & 0 & 0 & 0 & 0 & 0 & 0 \\
0 & 0 & 0 & 1 & -1 & 0 & 0 & 0 & 0 & 0 & 0 & 0 \\
0 & 0 & 0 & 0 & 0 & 0 & 1 & -1 & 0 & 0 & 0 & 0 \\
0 & 0 & 0 & 0 & 0 & 0 & 0 & 0 & 0 & 1 & -1 & 0 \\
x_{12} \\
x_{13} \\
x_{22} \\
x_{23} \\
x_{31} \\
x_{32} \\
x_{33} \\
x_{41} \\
x_{42} \\
x_{43}
\end{array}\right)
$$

The resulting modulation matrix is the following:

$$
\mathbf{X}=\frac{1}{2}\left(\begin{array}{ccc}
1.00 & 1.00 & 0.06 \\
1.00 & -0.14 & 0.99 \\
1.00 & -0.98 & 0.19 \\
1.00 & -0.06 & -1.00
\end{array}\right) \pm\left(\begin{array}{ccc}
0.00 & 0.01 & 0.17 \\
0.00 & 0.04 & 0.01 \\
0.00 & 0.01 & 0.06 \\
0.00 & 0.11 & 0.01
\end{array}\right)
$$


The associated demodulation matrix $\mathbf{X}^{\dagger}$, can then be obtained by inverting $\mathbf{X}$, obtained computing Eq. 11 pseudo-inverse. The resulting $\mathbf{X}^{\dagger}$ is shown in Eq. 12:

$$
\mathbf{X}^{\dagger}=\frac{1}{2}\left(\begin{array}{cccc}
1.09 & 0.88 & 0.91 & 1.12 \\
2.14 & -0.01 & -1.88 & -0.25 \\
0.21 & 1.85 & 0.08 & -2.14
\end{array}\right) \pm\left(\begin{array}{cccc}
0.00 & 0.00 & 0.00 & 0.00 \\
0.15 & 0.38 & 0.25 & 0.24 \\
0.20 & 0.34 & 0.03 & 0.31
\end{array}\right)
$$

Finally, Metis was thought as an externally occulted coronagraph. The highly vignetted aperture of the externally occulter coronagraph at lower heliocentric height compensates in part the high dynamic range, making possible the observation of the corona at higher heights. Anyway, to be able to see the real corona profile, a vignetting function (VF) for the visible channel, obtained from the average of four polarimetric flat field images at different polarization angle, were acquired in order to be applied to the in-flight VL images $m_{j}[\mathrm{DN} / \mathrm{pix}]$ (where $j=0,1,2,3$ is the polarization angle the image was taken with). The on-ground measured vignetting function is shown in Fig. 6. In detail, the dimensionless vignetting function VF is given from on ground calibration by:

$$
V F=\frac{\frac{1}{2} \sum_{i=0}^{3} M_{0 i} \varphi_{i}}{I_{0} \cdot\langle G\rangle \cdot\langle\eta\rangle \cdot P_{L} \cdot A_{T} \cdot T_{e x p}}
$$

where $\varphi_{i}$ are the polarimetric flat field images $[D N / p i x], M_{i j}$ the demodulation tensor elements and $I_{0}\left[\mathrm{ph} / \mathrm{s} / \mathrm{sr} / \mathrm{cm}^{2}\right]$ is the input radiance (obtained from the diode current used during calibration). The other parameters are: average gain $\langle G\rangle=0.119\left[\mathrm{DN} / \mathrm{e}^{-}\right]$, average efficiency $\langle\eta\rangle=0.118 \pm 0.002\left[\mathrm{e}^{-} / \mathrm{ph}\right]$ (inclusive of linear analyzer $1 / 2$ transmissivity and the average quantum efficiency), VL detector plate scale $P_{L}=2.5 \times 10^{-9}[\mathrm{sr} / \mathrm{pix}]$, entrance aperture area $A_{T}=12.6\left[\mathrm{~cm}^{2}\right]$ and exposure time $T_{\exp }[\mathrm{s}]$. In particular, the input radiance $I_{0}$ is obtained as:

$$
I_{0}=\frac{C_{d} / R}{h \nu} \frac{1}{A_{d} F o V_{d}} \frac{1}{T_{\text {filter }}}
$$

where are considered: diode current $C_{d}[\mathrm{~A}]$, responsivity $(@ 610 \mathrm{~nm}) R[\mathrm{~A} /(\mathrm{J} / \mathrm{s})]$, diode sensitive area $A_{d}\left[\mathrm{~cm}^{2}\right]$, filter transmittivity $T_{\text {filter }}$ and the diode field of view $F_{o} V_{d}$; obtained as:

$$
F_{o} V_{d}=2 \pi\left[1-\cos \left(\frac{\vartheta}{2}\right)\right]=2 \pi\left[1-\cos \left(\arctan \frac{r_{f}}{D_{f}}\right)\right]
$$

considering the filter-diode distance $D_{f}[\mathrm{~cm}]$ and the filter radius $r_{f}[\mathrm{~cm}]$.

The final VF was obtained as an average of $4 \mathrm{VFs}$, each one, with a fixed voltage and a different pre-polarizer orientation (the same procedure was performed keeping fixed the prepolarizer and changing the voltage of the LCVR obtaining a comparable result). This VF was then obtained with the theoretical demodulation tensor and the one obtained during on-ground calibration as well. Comparing them, the difference was at the order of $\sim 1 \%$. In order to keep "indipendent" the VF and the demodulation tensor, we choose to use the VF obtained using the theoretical $\mathbf{X}^{\dagger}$.

The UV vignetting function is the same of the VL channel, since visible and UV light share the same optical path in the telescope, which is the only vignetting optical element. However, some considerations need to be taken into account. In the next section we will explain more in detail how the VL vignetting function could be adapted to the UV channel.

\section{IN-FLIGHT CALIBRATION}

In this section, we are going to deal with analyzing, step by step, some of the main in-flight calibrations carried out during the first acquisitions performed by the Metis coronagraph (focusing, in particular, on the polarimeter functionality). More details on other aspects of in-flight calibrations will be presented in future works. 

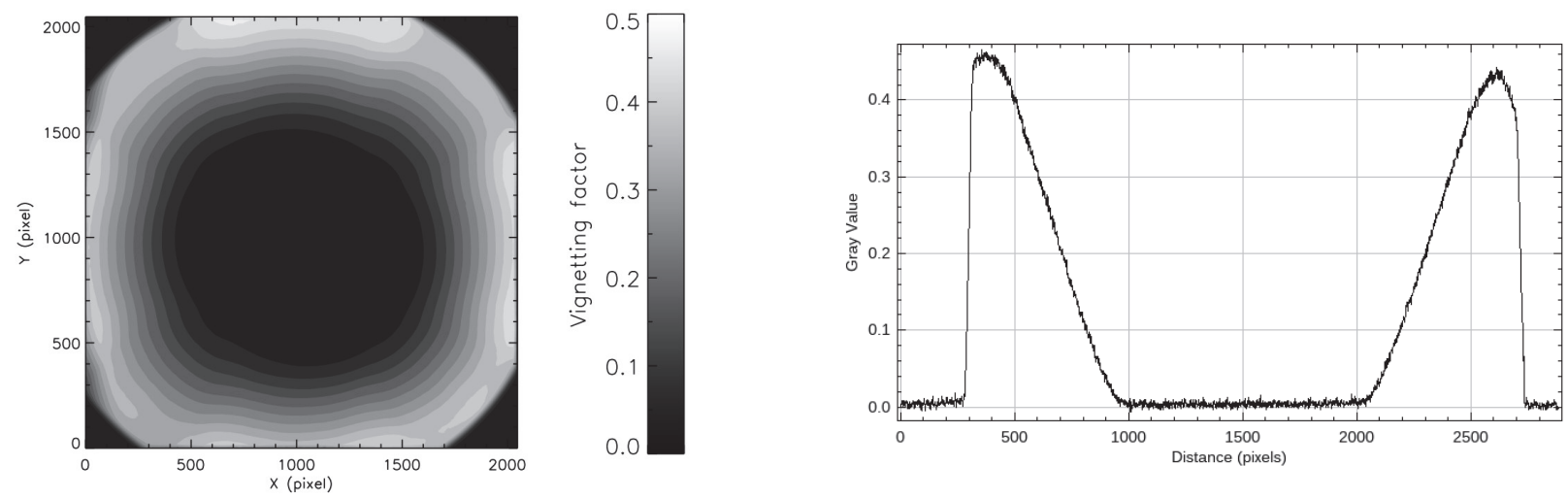

Figure 6: Metis vignetting function obtained during the on-ground calibration (on the left) and its diagonal cut, from $(0,0)$ to $(2047,2047)$, on the right.

\subsection{Vignetting function recentering}

Before discussing about different Metis in-flight calibration, a step took place before the actual calibration of the instrument. In particular, during the Metis first light, the images were dominated by stray light. That was due to a misalignment of the IO. This required an adjustment of the position of the IO in order to obtain a correct occultation of the solar disk (Fig. 8 - left). For this reason, also the VF acquired during the on-ground calibration required a "shift" of the same quantity in the same direction in order to be applied to flight images. To evaluate this shift, we have adopted a solution based on the use of the isophotes. In fact, it is possible to fit the points of equal brightness, near to the IO, using an ellipse or a circumference. Repeating this process for different brightness thresholds it is possible to obtain the IO center from the average of these fits output. By carrying out this process for the VF pre and post launch, can be possible to evaluate the IO shift. With the same procedure it is also possible to check on the in-flight position of the coronagraph field stop (FS) comparing its position before and after the launch (Fig. 7).

The VF can be shifted so that the "on-ground IO" coincides with the flying position. However, the internal occulter shift did not change the field stop and spiders position. A net shift of the entire vignetting would therefore have an over-/under- correction effect in the proximity to the external field of view. To avoid that, it was decided to stop for the amount of shifted vignetting function near the FS. This "partial shift" involves the creation of an "empty" area in which the data is missing; a "black belt" all around the frame inside the FS (Fig. 8 - right).

The values in this "black belt" are extrapolated trying to connect the internal and external values of this "empty" area. In first approximation, a linear function was considered for the data fitting (future adjustments can be performed by increasing the order of the fit). For each fit was considered data belonging to angle ranges of 1 degree (future adjustments can be made by decreasing the angle range step). An example of the linear fit extrapolation and the final VF after 360 fit (1 fit for each degree) are shown in Fig. 9.

The VL vignetting function can be used, under some considerations, for the UV images as well. In fact, considering the vignetting function obtained on ground for the VL channel, the UV one can be obtained by centering the on-ground VL IO with the center of the in-flight UV IO (both centers can be obtained using the isophothes). As shown before (Fig. 7), the on-ground VL IO center coordinates are: $(x, y)=(12 \pm 1,-62 \pm 1)$ with respect to the image center $(1023,1023)$. However, in order to use the visible VF as the UV VF, a necessary pre-step is to rebin the visible VF from $2048 \times 2048$ to $1024 \times 1024$. After the rebinning, the center of the onground visible IO is: $(x, y)=(7 \pm 1,-31 \pm 1)$ respect to the image center $(512,512)$. With the same method used to evaluate the VL IO center, it is possible to obtain the in-flight UV IO center. Summarizing the results, we have that the IO center for on-ground VL image $(1024 \times 1024)$ at $(x, y)=(7 \pm 1,-31 \pm 1)$ and the IO center for in-flight UV image $(1024 \times 1024)$ at $(x, y)=(-5 \pm 1,-3 \pm 1)$ both relative to the center of the image. Then, shifting the on-ground VL vignetting function of 12 pixels (from +7 to -5 ) to the left $(\mathrm{W} \rightarrow \mathrm{E})$ and 28 pixels (from -31 to -3 ) upward $(\mathrm{S} \rightarrow \mathrm{N}$ ) we obtain the in-flight UV vignetting function (Fig. 10). 

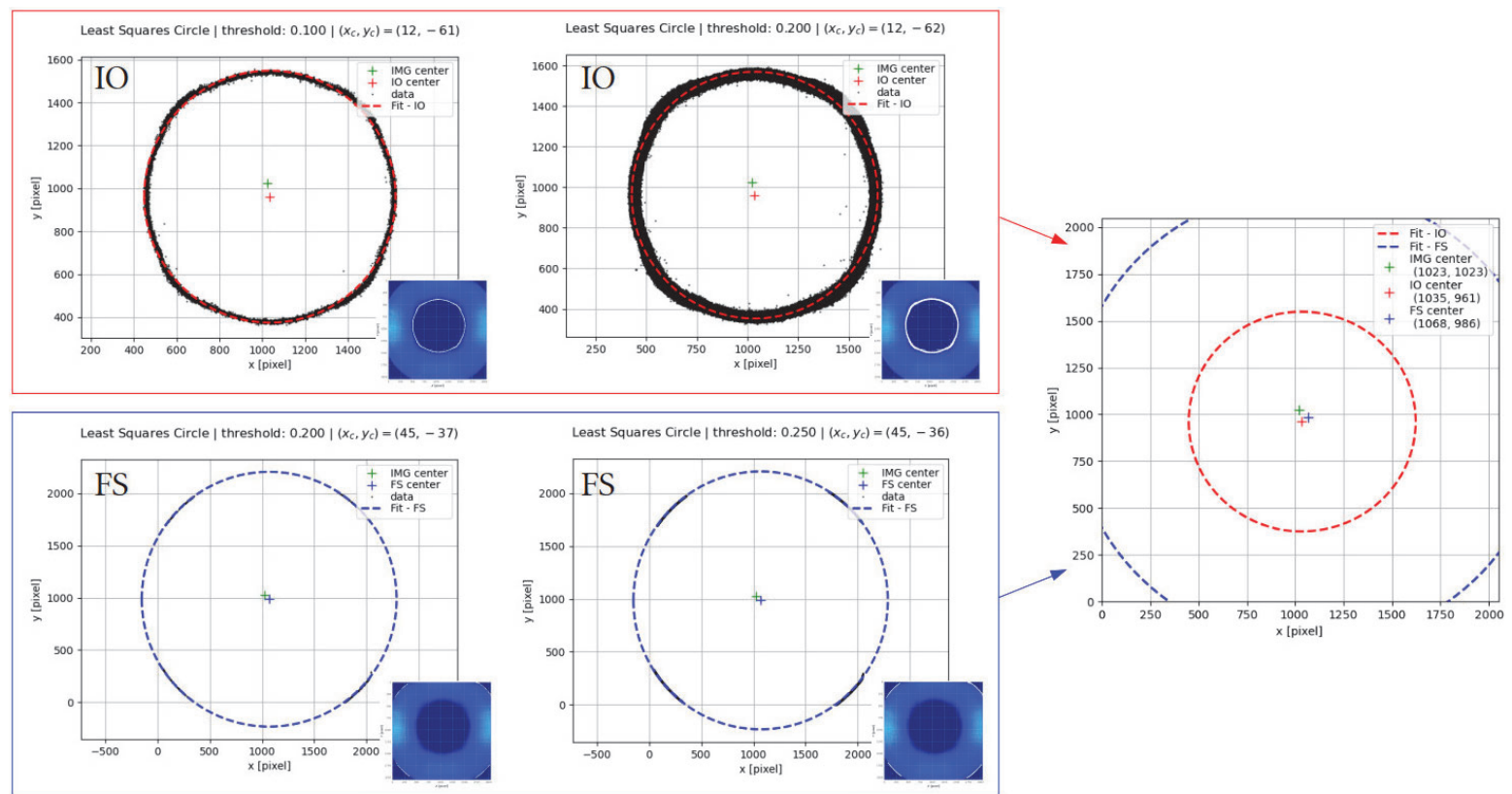

Figure 7: IO and FS center evaluation respect to the image center using isophotes with two different thresholds (indeed, the real final center -on the right- value is obtained by the average of 40 thresholds). In this partiular example we considerd on-ground VL vignetting function but the same procedure was performed also for in-flight VL and UV images in order to evaluate the respective pre- / post- launch shifts.
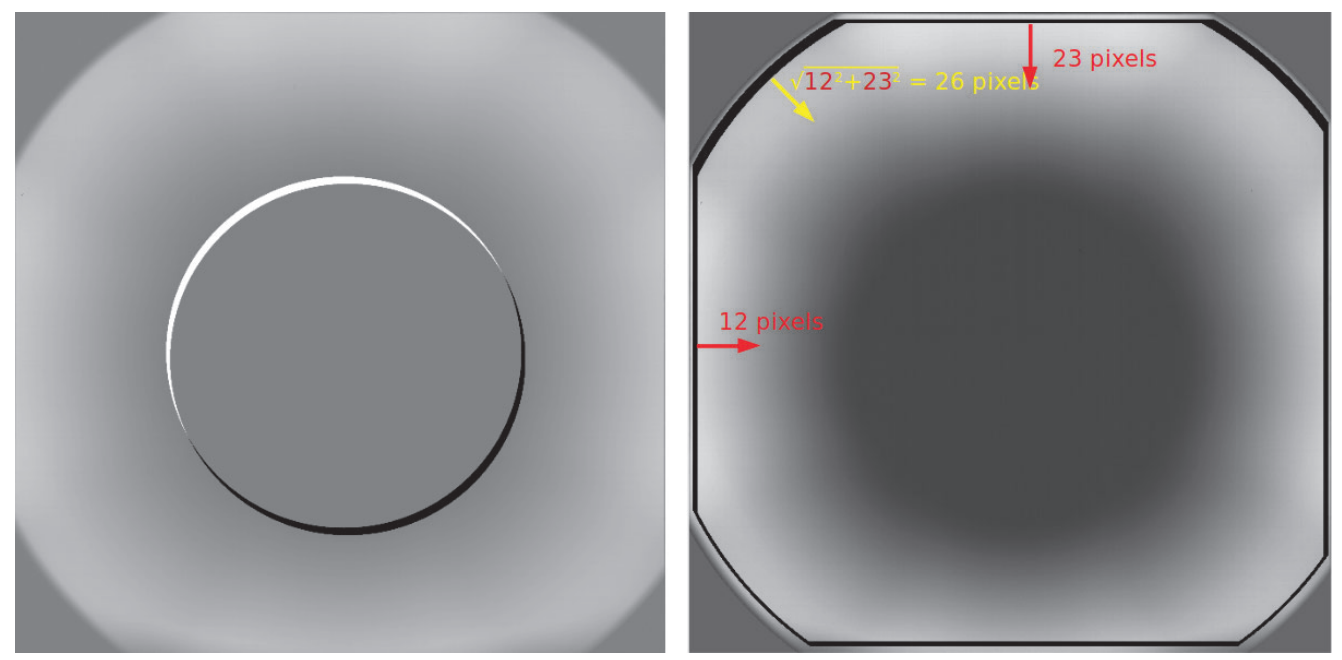

Figure 8: Left: IO center shift between on-ground VF (white occulter) and a post adjustment in-flight VF (black occulter). It is possible to see that this shift seems to be almost along a spider. Right: VL vignetting function shifted of $d=26$ pixels to obtain the pre- / post- launch IO center recentering. This shift was not applied to the VF areas near to the FS (black belt).

\subsubsection{Polarized Brigthness}

After the in-flight IO adjustment, it was possibile to obtain images not dominates by stray-light and test the new, recentered, VF on them. In Fig. 11 it is shown an example of polarized brightness (pB) obtained as $\sqrt{Q^{2}+U^{2}}$. The Q and U Stokes parameters come from the 4 images of the nominal quadruplet (which were subtracted for the dark, divided by the flat field and corrected by the recentered vignetting function). 

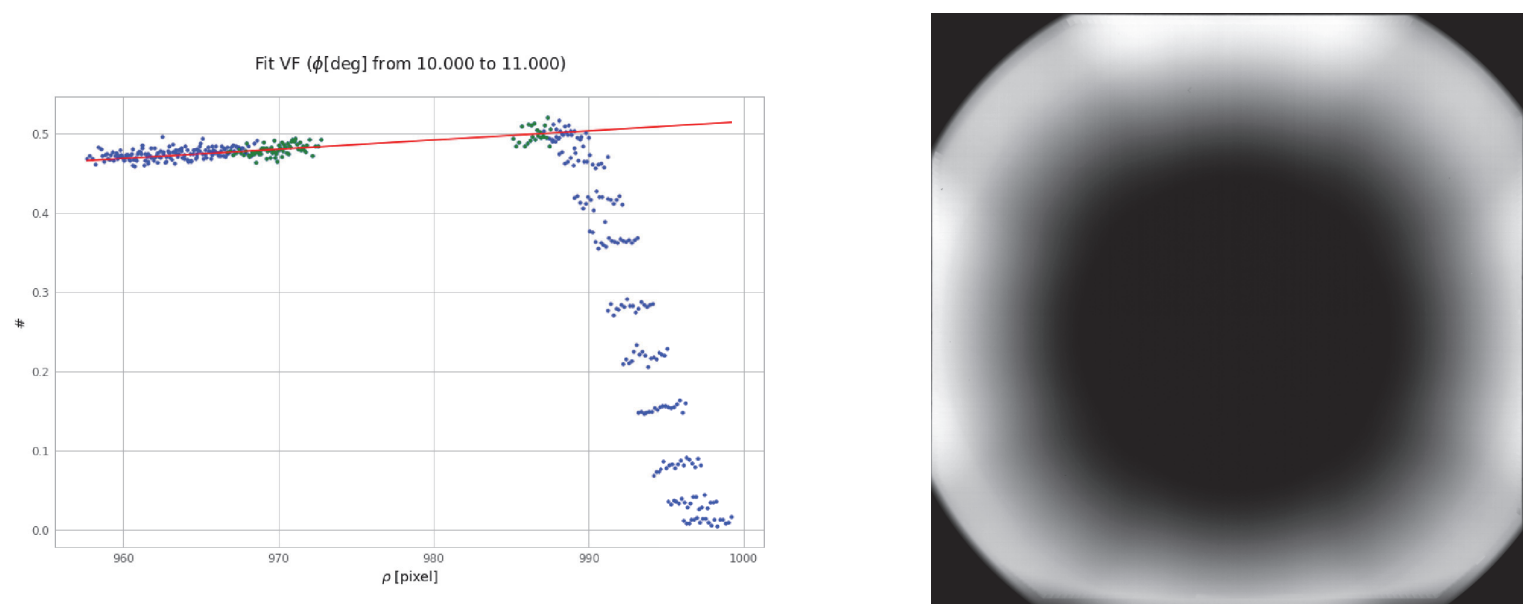

Figure 9: Left: An example of linear fit (for the angle range $11^{\circ}-12^{\circ}$ ). The blue dots are the vignetting function radial values in the considered angle range and the green ones are the points considered for the fit extrapolation (the red line). Right: VF after in-flight IO recentering.

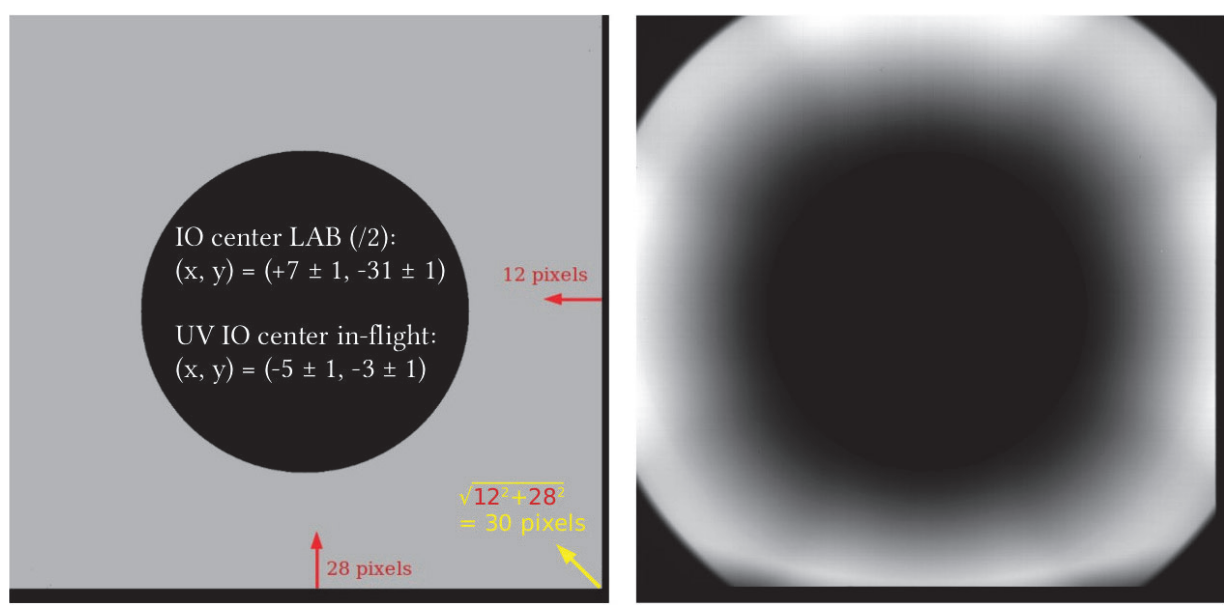

Figure 10: Left: VF for UV channel. It was obtained through the shift of VL vignetting function to recenter the UV and VL IO centers. The black pixels on side of the frame, are due to this shift (SW $\rightarrow$ NE direction). Right: VF for UV channel obtained after the VL vignetting function shift.

It is possible to compare the $\mathrm{pB}$ value along a radial distance as measured by Metis with the $\mathrm{pB}$ obtained, the same day, by other instruments (Fig. 11). In particular, we compared the Metis, LASCO C2, LASCO-C3 and K-Cor pB of May 15 ${ }^{\text {th }}, 2020$ because, at that time, Solar Orbiter was almost aligned with the Sun-Earth axis. As it is possible to see, the Metis $\mathrm{pB}$ values are consistent with the other instruments $\mathrm{pB}$. This result is also a symptom of a good radiometric calibration.

\subsection{Polarized flat field}

During IT-7 campaign (carried out on June $8^{\text {th }}$; distance to the Sun: 0.52 A.U.) a complete roll was performed by SolO S/C. During the roll maneuver, the Metis polarimeter acquired several sets of images of the K-corona polarized brightness for a total of 8 roll positions (Fig. 12). The goal is to check the polarized flat-field: the pB image of the same coronal structure was measured in different locations of the detector focal plane. The comparison of these images will give indications of the differences in the polarimetric response from different locations across the polarimeter and the detector. To perform this study, we considered the Degree of Linear Polarization (DoLP) obtained dividing the pB for the first Stokes parameter I. 

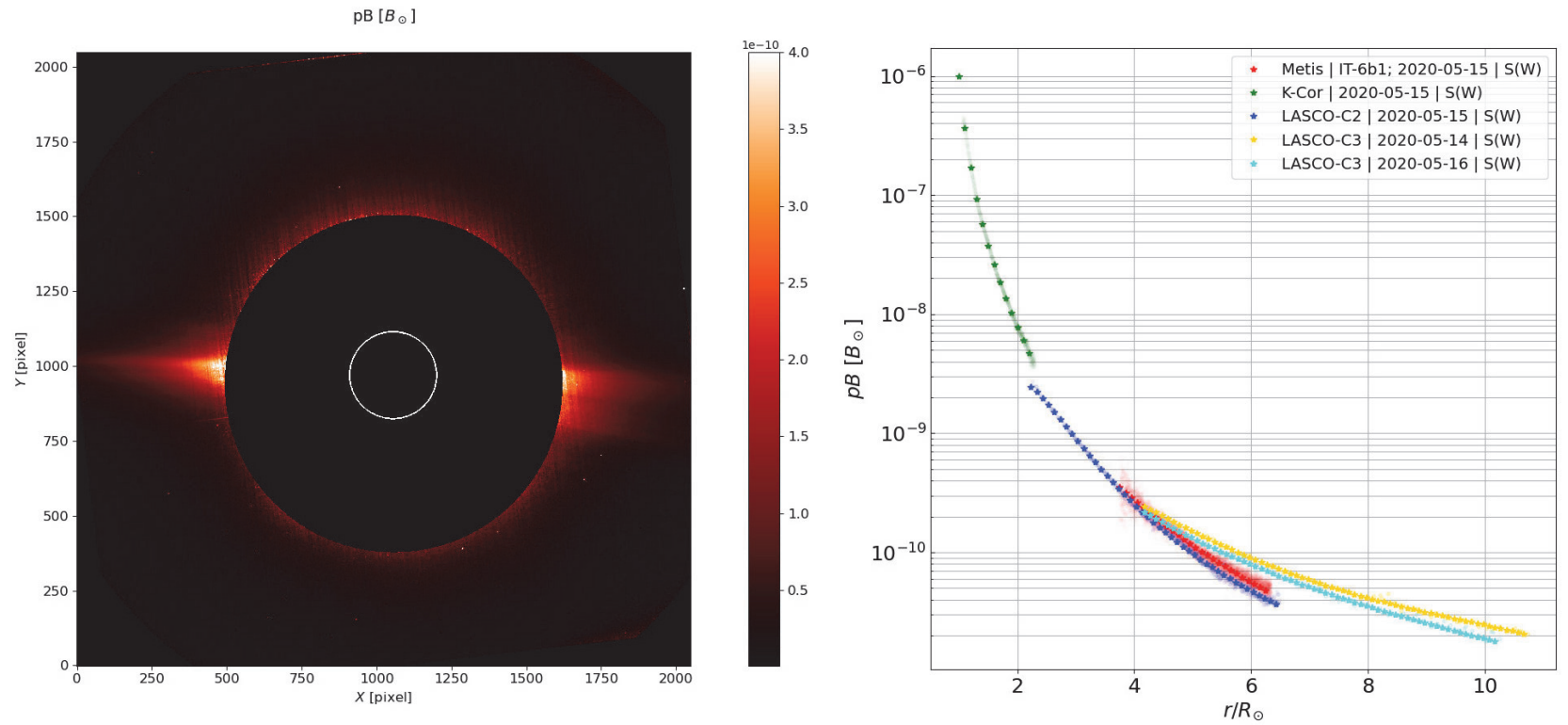

Figure 11: Left: An example of Metis polarized brightness (pB). Right: Comparison of $\mathrm{pB}$ values along the West streamer between Metis, LASCO and K-Cor instruments during an almost Sun-S/C-Earth alignment.

$\mathrm{pB} /\left(\right.$ roll n. $\left.0,0^{\circ}\right)$

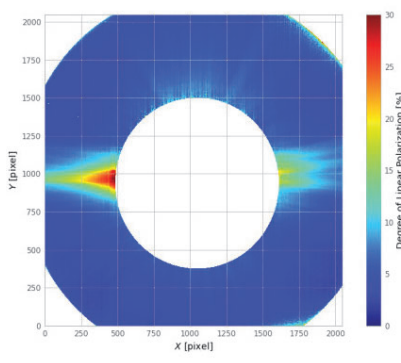

$\mathrm{pB} /\left(\mathrm{rol} \mid \mathrm{n} .4,180^{\circ}\right)$

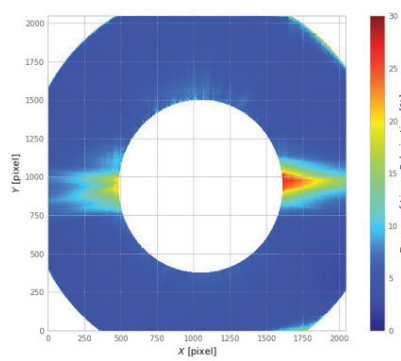

$\mathrm{PB} /$ (roll n.1. 45

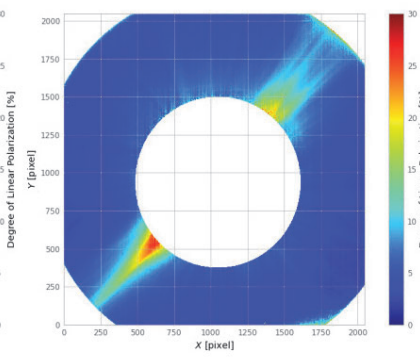

PBII (roll . .5, 225)

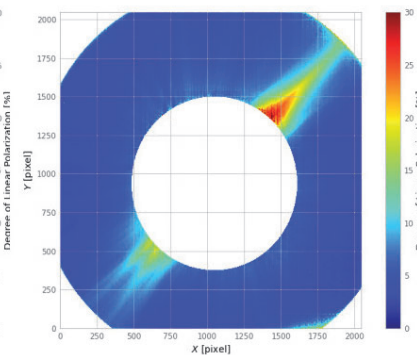

$\mathrm{pB/I}$ (roll n.2, $90^{\circ}$

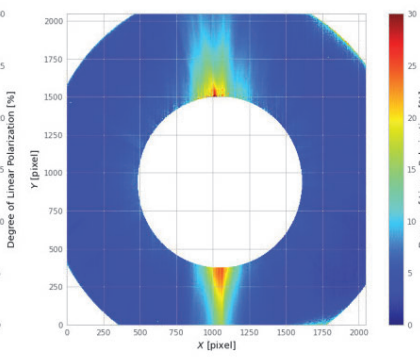

PBII (roll n.6, 270 )

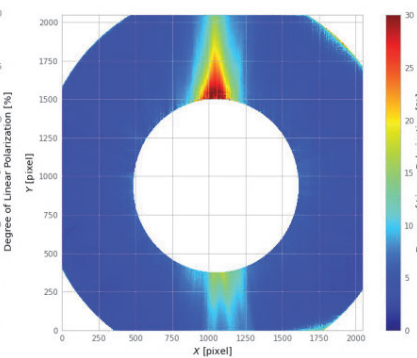

$\mathrm{pB/} /\left(\right.$ roll n. $\left.3,135^{\circ}\right)$

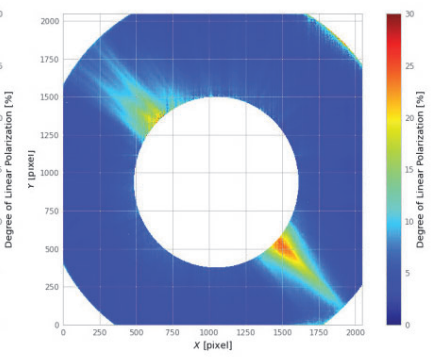

$\mathrm{pB} /\left(\right.$ roll $\left..7,315^{\circ}\right)$

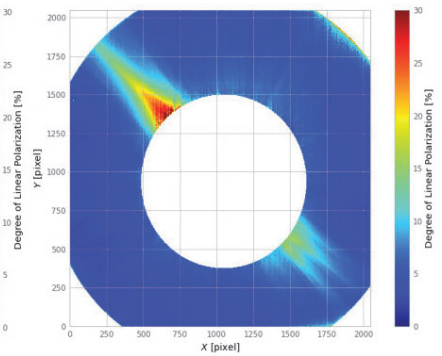

Figure 12: Degree of linear polarization (pB/I) of the K-corona for the 8 different $\mathrm{S} / \mathrm{C}$ roll positions.

Quantitatively, we can plot the value of the $\mathrm{pB} / \mathrm{I}$ variation for each roll in a fixed region on the frame (for example along a streamer). Considering this region and following it the $\mathrm{S} / \mathrm{C}$ rotation during the 8 different rolls (Fig. 13) maintaining the fixed distance from the Sun center (found by astrometry), we expect to find almost the same values of $\mathrm{pB} / \mathrm{I}$.

Fig. 14 shows the result. The $\mathrm{pB} / \mathrm{I}$ values are consistent with the expected values (i.e. the $\mathrm{pB} / \mathrm{I}$ value at the same heliocentric height is almost constant for different roll). The average value is: $p B / I=0.25 \pm 0.01$ with an averaged percentage error $\sim 4 \%$ (all the values are reported in 3). In particular, the dispersion of the $\mathrm{pB} / \mathrm{I}$ mean 

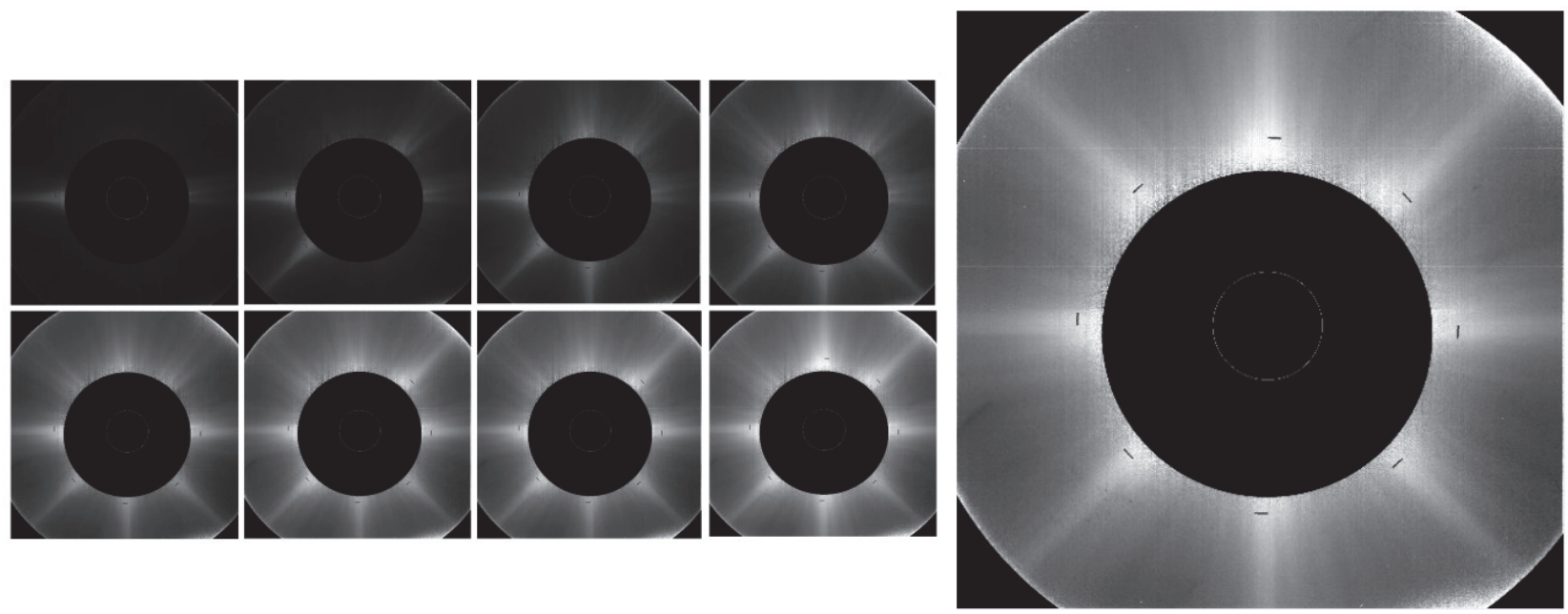

Figure 13: Considered region (black rectangles) during the 8 rolls. The white circle inside the internal occulter show the Sun size and position.

values using the theoretical demodulation tensor is higher $(p B / I=0.21 \pm 0.02$ with an averaged percentage error $\sim 10 \%$ ) than using the demodulation tensor obtained during the on-ground calibration. These results confirm the value of using the demodulation tensor obtained from the ground-based calibration. With the theoretical demodulation tensor there is an accuracy on the $\mathrm{pB} / \mathrm{I}$ values of about $10 \%$ (whereas a $\sim 3 \%$ is due to the dispersion of the $\mathrm{pB} / \mathrm{I}$ in the considered area) instead of a $4 \%$ accuracy obtained with the demodulation tensor from on-ground calibration (with a $\sim 2 \%$ due to the dispersion). Then, in conclusion, by using the demodulation tensor from ground calibration the accuracy in the polarization measurement improves by a factor of $\sim 4$.
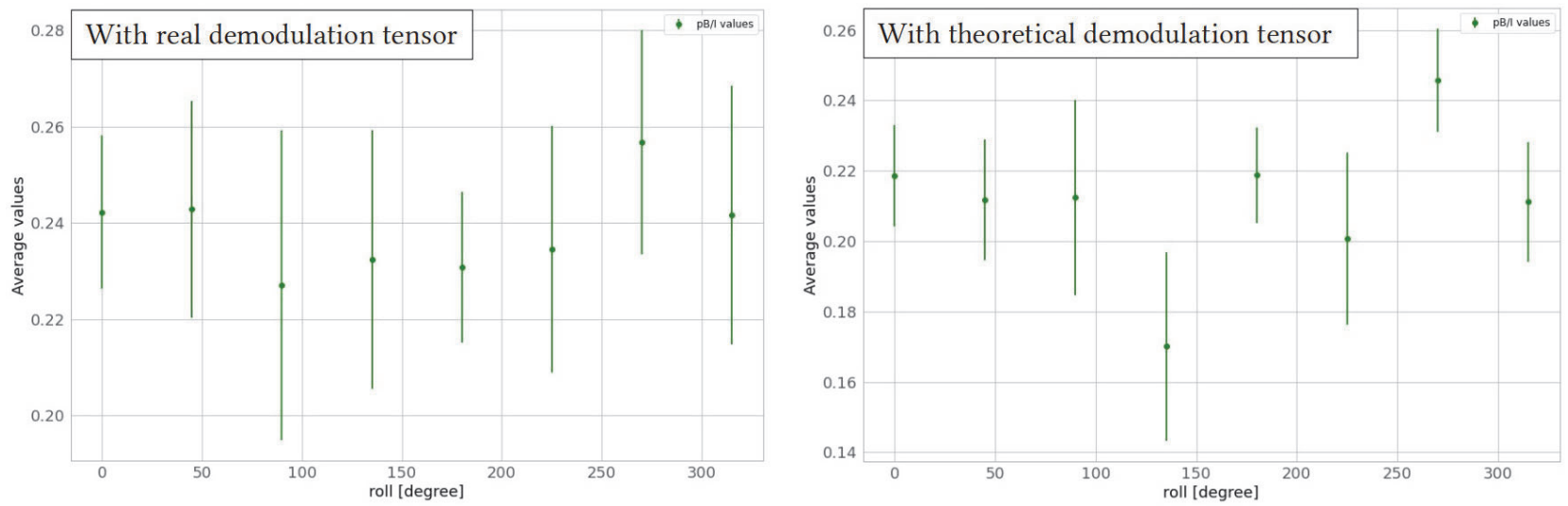

Figure 14: pB/I average over the pixels inside the selected regions (for each roll) using the demodulation tensor obtained during the ground calibration (on the left) and with a theoretical one (on the right). The bars represent the dispersion inside the considered area.

The same kind of study can be performed using the quantity Q/I and U/I instead of $\mathrm{pB} / \mathrm{I}$. What we expect to obtain is a Malus curve behaviour with a shift between them of $\sim 45^{\circ}$. Plotting these quantities, we obtain the expected trend for both Q/I and U/I (Fig. 15). 
Table 3: pB/I average values with standard deviation (pB and I obtained through the on-ground demodulation tensor [left] and theoretical one [right]). Dispersion column contains the standard deviation obtained considering the $\mathrm{pB} / \mathrm{I}$ values in the region at fixed heliocentric distance (black squares in Fig. 13) for each roll.

\begin{tabular}{|ccc|ccc|}
\hline Roll & $\begin{array}{c}\text { On ground } X^{\dagger} \\
\mathbf{p B} / \mathbf{I}\end{array}$ & Dispersion & Roll & $\begin{array}{c}\text { Theoretical } X^{\dagger} \\
\mathbf{p B} / \mathbf{I}\end{array}$ & Dispersion \\
\hline 0 & 0.242 & 0.016 & 0 & 0.219 & 0.014 \\
1 & 0.243 & 0.023 & 1 & 0.212 & 0.017 \\
2 & 0.227 & 0.032 & 2 & 0.212 & 0.028 \\
3 & 0.232 & 0.027 & 3 & 0.170 & 0.027 \\
4 & 0.231 & 0.016 & 4 & 0.219 & 0.014 \\
5 & 0.235 & 0.026 & 5 & 0.201 & 0.024 \\
6 & 0.257 & 0.023 & 6 & 0.246 & 0.015 \\
7 & 0.242 & 0.027 & 7 & 0.211 & 0.017 \\
\hline Average: & 0.239 & 0.024 & Averge: & 0.211 & 0.019 \\
StDev: & 0.009 & 0.006 & StDev: & 0.021 & 0.006 \\
\hline StDev/Average: & $4 \%$ & $2 \%$ & StDev/Average: & $10 \%$ & $3 \%$ \\
\hline
\end{tabular}

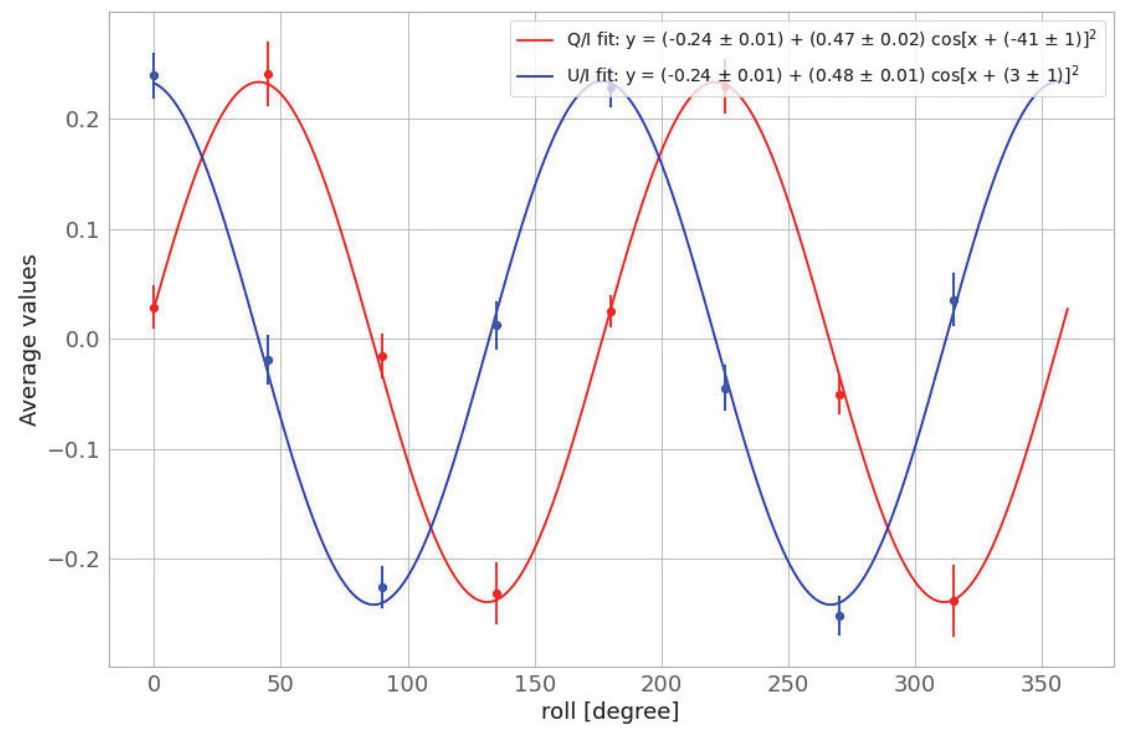

Figure 15: Q/I and U/I averages over the pixels inside the selected regions (for each roll) using the demodulation tensor obtained during the on-ground calibration.

\subsection{In-flight calibration of the LCVR}

\subsubsection{Method 1}

Considering the geometric properties of the linearly polarized light in the K-corona (Fig. 16) it is possible to perform an in-flight calibration of the LCVR retardances. It is necessary to consider 4 corona images at different linear polarizations. In particular, considering the Stokes vector in the reference system of the image, for a point $\mathrm{P}$ with coordinates $(r, \alpha)$, we can rewrite the recorded signal for each sensor element such as: ${ }^{13}$

$$
\frac{2 m_{i}-I}{p B}=-\cos \left(2 \alpha-\delta_{i}\right)
$$


where $m_{i}$ are the single images of a quadruplet, I is the first Stokes parameter and $\delta_{i}$ are the retardance values that can be estimated through the 3-parameters regression:

$$
y_{i}=P_{0}-P_{1} \cos \left(2 x-P_{2}\right)
$$

considering $y_{i}=\left(2 m_{i}-I\right) / p B, P_{0}$ is the bias of fitting curve, $P_{1}$ is the altitude modulation and $P_{2}=\delta_{i}$.

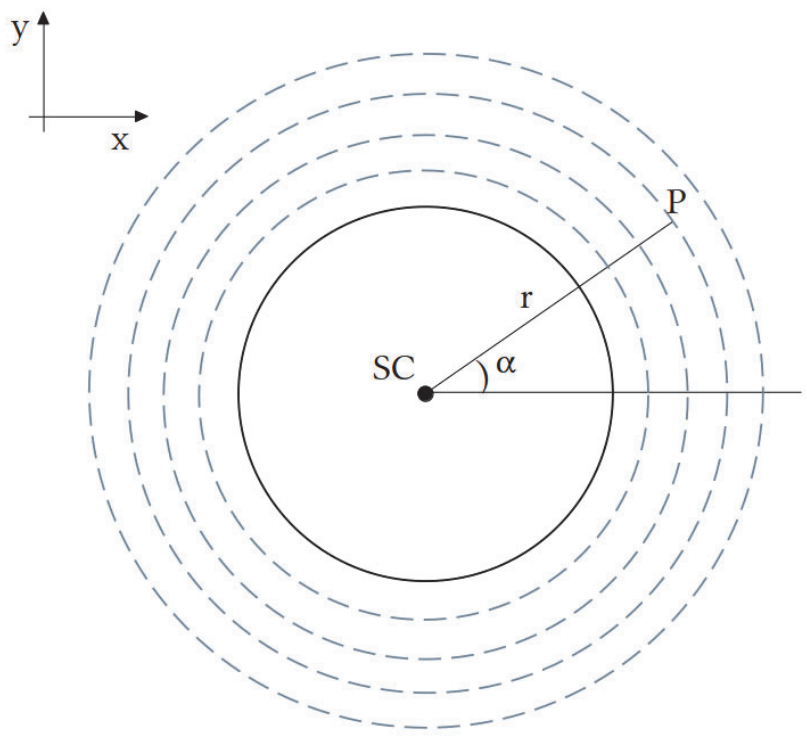

Figure 16: Direction of K-corona linear polarization (tangent to the solar limb) for different distance from the Sun center (SC).

Obtained the Sun center (SC) position by astrometry, it is possible to perform the 4 regressions (i.e. one for each $y_{i}$ obtained from the respective image of the quadruplet $m_{i}$ ), for a fixed distance from the center of the Sun. What we obtain is shown in Fig. 17. It is possible to see that in some regions the error bars are much higher than elsewhere. This is due to the fact that the error is proportional to $\sim 1 / p B$. For this reason, the less noisy areas are those with the most polarized signal (along the streamers). The reduced $\chi^{2}$ is always $<1$ (oscillating between values of 0.3 and 0.6 ). Looking at the $P_{2}$ parameters, we can obtain the polarization angles $\vartheta$. In particular, we performed this study for different heliocentric heights. We considered 10 distances from 580 to 670 pixels from the Sun center in order to avoid noisy regions too near or too far away from the IO edges. The expected values from ground calibration for the nominal quadruplet polarization angles are: $\left(49.1^{\circ}, 84.3^{\circ}\right.$, $133.2^{\circ}, 181.1^{\circ}$ ) with an error or $1.4^{\circ}$ each. From the average of the values at different heliocentric heights, we obtain: $\left(45.0^{\circ} \pm 0.1^{\circ}, 81.4^{\circ} \pm 0.1^{\circ}, 128.7^{\circ} \pm 0.1^{\circ}, 175.4^{\circ} \pm 0.1^{\circ}\right)$ that are consistent with the expected ones.

\subsubsection{Method 2}

It is possible to obtain an in-flight calibration of the LCVR using data from the IT-7 rolls as well. In fact, considering always the same region in the solar corona, the S/C rotation gives the same result as "rotate the polarizer". We therefore expect that the recorded intensities follow the Malus's law (Fig. 18). By making a fit with this function we can have an estimation of the delays and compare them with those expected from the ground calibration and what we obtained in the previous method (Tab. 4). What we obtain is: $\left(46^{\circ} \pm 4^{\circ}, 82^{\circ} \pm 4^{\circ}\right.$, $\left.130^{\circ} \pm 5^{\circ}, 176^{\circ} \pm 5^{\circ}\right)$ that are again consistent with the expected one. 


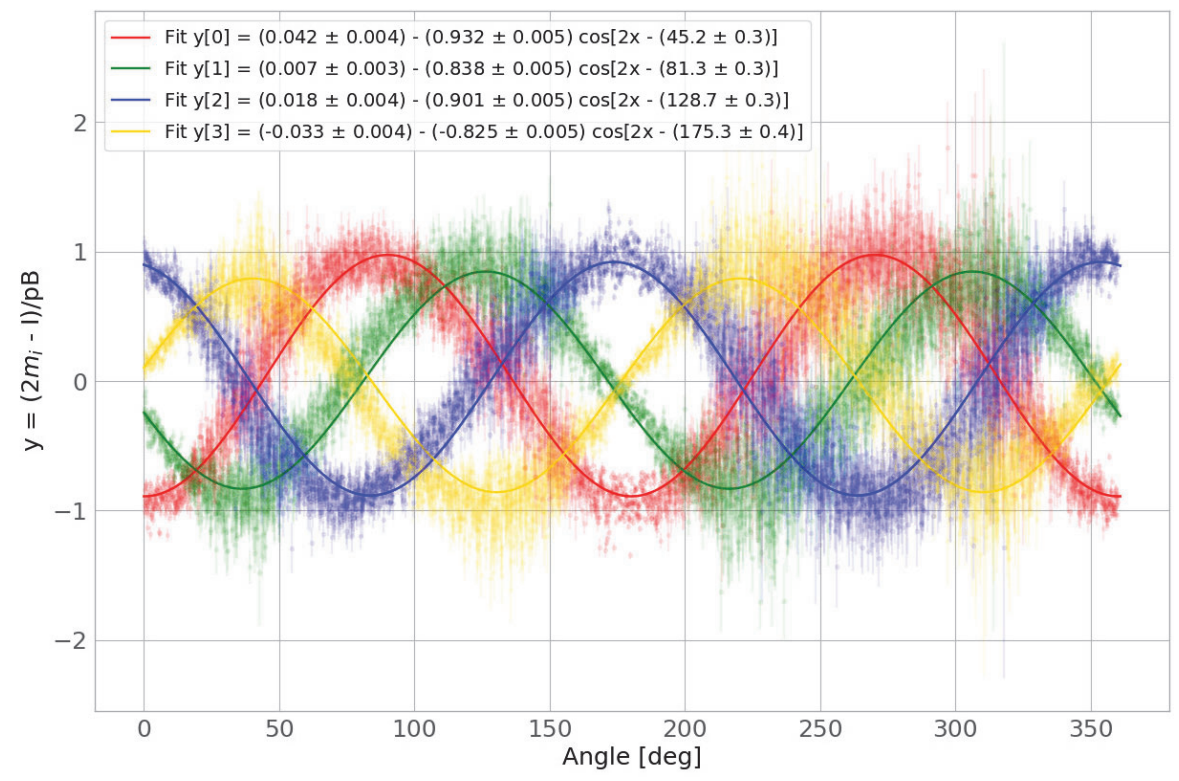

Figure 17: Plot of the 4 regressions to perform the in-flight calibration of the LCVR retardances. The considered data come from a quadruplet acquired during the "roll n.0" of the IT-7 campaign (see Fig. 12) for a fixed heliocentric height (620 pixels from the Sun center in this particular case).

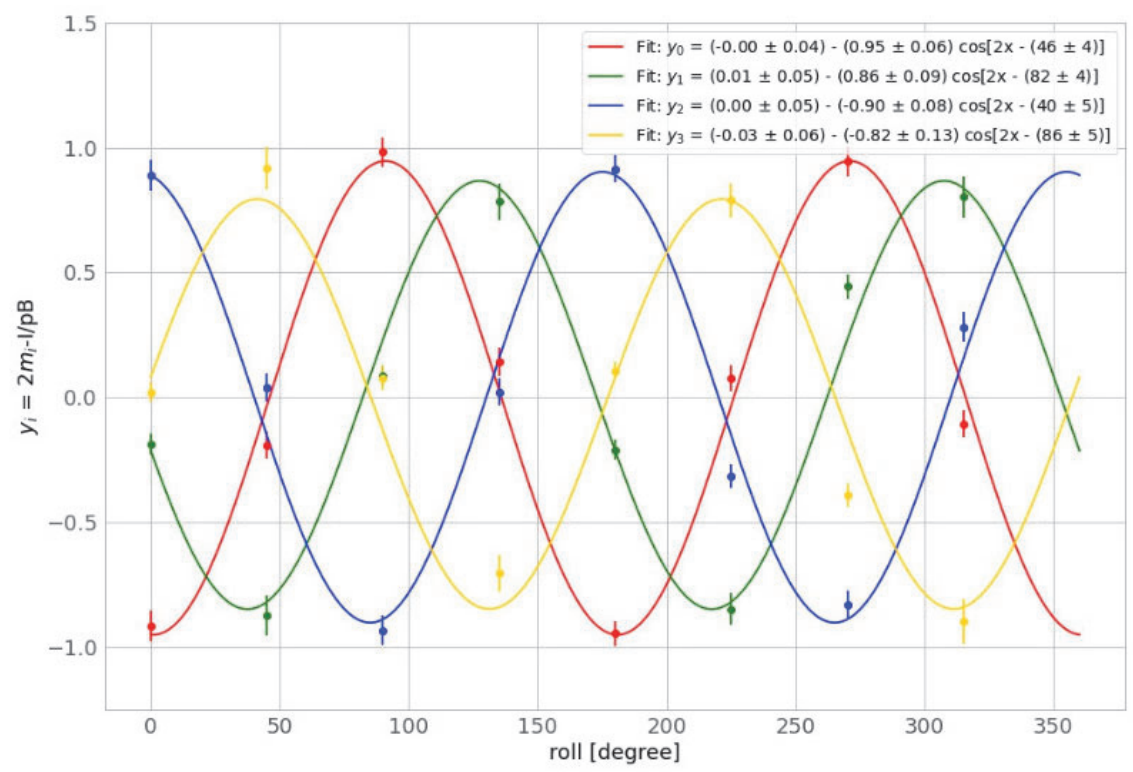

Figure 18: Malus curve obtained from $y_{i}=\left(2 m_{i}-I\right) / p B$ average values over the pixels in a fixed region in the solar corona during each roll.

\subsection{Different quadruplets}

During the first remote sensing checkout window (RSCW1 - June $18^{\text {th }}, 2020$ - distance to the Sun: 0.52 A.U.) campaign, polarimetric sequences with different quadruplets of PMP voltages were acquired. The goal is to check the polarimeter's response for different voltage configurations. All these quadruplets consist of 4 polarization angles separated by $\sim 45^{\circ}$ from each other. All the considered quadruplets, with an effective angle error of $\sim 1^{\circ}$, are summarized in Tab. 5 . 
Table 4: Comparison between the LCVR polarization angles from on-ground and in-flight calibration.

\begin{tabular}{|c|c|c|}
\hline On-ground & In-flight [method 1] & In-flight [method 2] \\
\hline $49^{\circ} \pm 1^{\circ}$ & $45.0^{\circ} \pm 0.1^{\circ}$ & $46^{\circ} \pm 4^{\circ}$ \\
$84^{\circ} \pm 1^{\circ}$ & $81.4^{\circ} \pm 0.1^{\circ}$ & $82^{\circ} \pm 4^{\circ}$ \\
$133^{\circ} \pm 1^{\circ}$ & $128.7^{\circ} \pm 0.1^{\circ}$ & $130^{\circ} \pm 5^{\circ}$ \\
$181^{\circ} \pm 1^{\circ}$ & $175.4^{\circ} \pm 0.1^{\circ}$ & $176^{\circ} \pm 5^{\circ}$ \\
\hline
\end{tabular}

Table 5: Comparison between the LCVR polarization angles from on-ground and in-flight calibration.

\begin{tabular}{|c|ccc|}
\hline Quadruplet & Effective angle $\left[ \pm 1^{\circ}\right]$ & Applied voltage $[\mathrm{mV}]$ & Parameter label \\
\hline 1 & $49.1^{\circ}, 84.3^{\circ}, 133.2^{\circ}, 181.1^{\circ}$ & $15837,10048,7318,6051$ & $35,65,105,145$ \\
2 & $17.0^{\circ}, 60.7^{\circ}, 108.9^{\circ}, 157.6^{\circ}$ & $30583,13216,8344,6597$ & $5,45,85,125$ \\
3 & $37.3^{\circ}, 96.4^{\circ}, 145.5^{\circ}, 194.6^{\circ}$ & $19573,9087,6924,5810$ & $25,75,115,155$ \\
4 & $24.6^{\circ}, 72.4^{\circ}, 121.1^{\circ}, 169.4^{\circ}$ & $25362,11359,7776,6313$ & $15,55,95,135$ \\
\hline
\end{tabular}

Using the demodulation tensor associated with each quadruplet (obtained during on ground calibration), it is possible to get the corresponding $\mathrm{pB}$ (in particular, $5 \mathrm{pB}$ were obtained; two acquisitions with "quadruplet 4" were performed). Comparing them, we expect to not observe huge variations. To check that, we considered 4 different $\mathrm{pB}$ regions (along streamers, coronal holes, etc...) and we compared the average of the pixels inside them (Fig. 19). We obtain that the differences between the $\mathrm{pB}$ are $\leq 2 \%$.

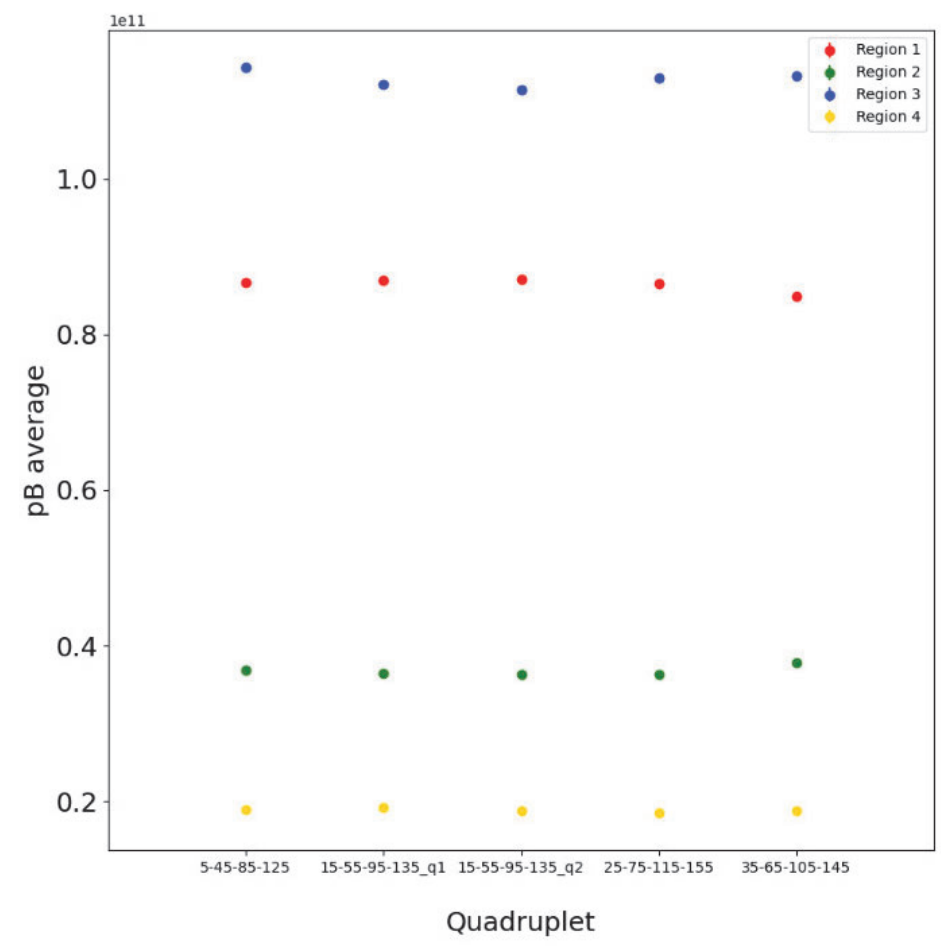

Figure 19: Comparison between the average of the four $\mathrm{pB}$ regions for the different quadruplets. The differences between the different $\mathrm{pB}$ are less than $2 \%$. 


\section{CONCLUSION}

This paper reports the prelminary in-flight calibration of the polarimeter of the Metis coronagraph on board Solar Orbiter. The VF obtained during on-ground calibration was applied to the in-flight images after an in-flight IO adjustment. The VL vignetting function was adapted for use in the UV channel. The polarimetric flat field was verified with in-flight polarimeter calibrations. This was carried out by changing the applied tension to the LCVR or using a fixed tension during a roll maneouver of the space-craft. The results are consistent with the on-ground calibration. The comparison of the $\mathrm{pB}$ resulting from quadruplets with different applied tensions to the polarimeter LCVR yielded differences within $\leq 2 \%$.

\section{ACKNOWLEDGMENTS}

This paper has been possible thanks to the whole Metis team and the contributions of the many listed authors. Among the others, a particular acknowledgment is due to E. Antonucci, who led this Project as Principal Investigator until the delivery of the instrument to ESA in September 2017, when M. Romoli took over this role.

This work has been supported by the Italian Space Agency - ASI. Metis team thanks the former PI, Ester Antonucci, for leading the development of Metis until the final delivery to ESA. The industrial contractor for the Metis project has been a temporary consortium between OHB Italia (for opto-mechanical design, electronics and the software) and Thales Alenia Space Italia (for telescope thermal and structural design and realization, application software, instrument integration, alignment and test). The primary and secondary mirrors were provided as Czech contribution to Metis; the mirror hardware development and manufacturing was possible thanks to the Czech PRODEX Programme. The authors thank also ALTEC Company for providing logistic and technical support.

\section{REFERENCES}

[1] Müller, D., St. Cyr, O. C., Zouganelis, I., Gilbert, H. R., Marsden, R., Nieves-Chinchilla, T., Antonucci, E., Auchère, F., Berghmans, D., Horbury, T. S., and et al., "The solar orbiter mission," Astronomy $\mathcal{E}$ Astrophysics 642, A1 (Sep 2020).

[2] Fineschi, S., Antonucci, E., Gardiol, D., da Deppo, V., Naletto, G., Romoli, M., Cacciani, A., and Malvezzi, M., "Extended UV corona imaging from the Solar Orbiter: the Ultraviolet and Visible-light Coronagraph (UVC)," in [Solar encounter. Proceedings of the First Solar Orbiter Workshop], Battrick, B., SawayaLacoste, H., Marsch, E., Martinez Pillet, V., Fleck, B., and Marsden, R., eds., ESA Special Publication 493, 217-222 (Sept. 2001).

[3] Inhester, B., "Thomson scattering in the solar corona," arXiv: Solar and Stellar Astrophysics v2, 104 (2016).

[4] Raouafi, N. E., "Coronal Polarization," in [Solar Polarization 6], Kuhn, J. R., Harrington, D. M., Lin, H., Berdyugina, S. V., Trujillo-Bueno, J., Keil, S. L., and Rimmele, T., eds., Astronomical Society of the Pacific Conference Series 437, 99 (Apr. 2011).

[5] Van De Hulst, H. C., "The electron density of the solar corona," B.A.N. 11, 135 (Feb. 1950).

[6] Fineschi, S., Naletto, G., Romoli, M., and et al., "Optical design of the multi-wavelength imaging coronagraph metis for the solar orbiter mission," Experimental Astronomy 49 (May 2020).

[7] Antonucci, E., Romoli, M., Andretta, V., Fineschi, S., Heinzel, P., Moses, J. D., Naletto, G., Nicolini, G., Spadaro, D., Teriaca, L., and et al., "Metis: the solar orbiter visible light and ultraviolet coronal imager," Astronomy \&3 Astrophysics 642, A10 (Sep 2020).

[8] Fineschi, S., Zangrilli, L., Rossi, G., Gori, L., Romoli, M., Corti, G., Capobianco, G., Antonucci, E., and Pace, E., "KPol: liquid crystal polarimeter for K-corona observations from the SCORE coronagraph," in [Solar Physics and Space Weather Instrumentation], Fineschi, S. and Viereck, R. A., eds., Society of Photo-Optical Instrumentation Engineers (SPIE) Conference Series 5901, 389-399 (Aug. 2005).

[9] Zangrilli, L., Fineschi, S., and Capobianco, G., "Calibration of the EKPol K-corona imaging polarimeter," in [Solar Physics and Space Weather Instrumentation III], Fineschi, S. and Fennelly, J. A., eds., Society of Photo-Optical Instrumentation Engineers (SPIE) Conference Series 7438, 74380W (Aug. 2009). 
[10] Alvarez-Herrero, A., Uribe-Patarroyo, N., Parejo, P. G., Vargas, J., Heredero, R. L., Restrepo, R., MartínezPillet, V., del Toro Iniesta, J. C., López, A., Fineschi, S., Capobianco, G., Georges, M., López, M., Boer, G., and Manolis, I., "Imaging polarimeters based on liquid crystal variable retarders: an emergent technology for space instrumentation," in [Polarization Science and Remote Sensing V], Shaw, J. A. and Tyo, J. S., eds., 8160, 312 - 329, International Society for Optics and Photonics, SPIE (2011).

[11] Casti, M., Fineschi, S., and et al., "Calibration of the liquid crystal visible-light polarimeter for the Metis/Solar Orbiter coronagraph," in [Space Telescopes and Instrumentation 2018: Optical, Infrared, and Millimeter Wave], Lystrup, M., MacEwen, H. A., Fazio, G. G., Batalha, N., Siegler, N., and Tong, E. C., eds., 10698, 930 - 943, International Society for Optics and Photonics, SPIE (2018).

[12] Casti, M., Fineschi, S., and et al., "Metis/Solar Orbiter polarimetric visible light channel calibration," in [International Conference on Space Optics - ICSO 2018], Sodnik, Z., Karafolas, N., and Cugny, B., eds., 11180, 1255 - 1269, International Society for Optics and Photonics, SPIE (2019).

[13] Elmore, D. F., Card, G. L., Lecinski, A. R., Lites, B. W., Streander, K. V., and Tomczyk, S., "Calibration procedure for the polarimetric instrument for Solar Eclipse-98," in [Instrumentation for UV/EUV Astronomy and Solar Missions], Fineschi, S., Korendyke, C. M., Siegmund, O. H., and Woodgate, B. E., eds., Society of Photo-Optical Instrumentation Engineers (SPIE) Conference Series 4139, 370-377 (Dec. 2000). 